\title{
EGb in the Treatment for Patients with VCI: A Systematic Review and Meta-Analysis
}

\author{
Min Zhan ${ }^{1 D},{ }^{1,2,3}$ Linjuan Sun, ${ }^{1}$ Jianxun Liu, ${ }^{1}$ Zixiu Zeng, ${ }^{4}$ Wei Shen, ${ }^{1}$ Huimin Li, ${ }^{2}$ \\ Ying Wang, ${ }^{4}$ Fuhua Han, ${ }^{4}$ Jingzi Shi, ${ }^{4}$ Xinyun Zeng, ${ }^{4}$ Xiyue Lu, ${ }^{4}$ Yunling Zhang ${ }^{\mathbb{D}}{ }^{1}$ \\ and Xing Liao $\mathbb{D}^{2}$ \\ ${ }^{1}$ Xiyuan Hospital, Chinese Academy of Traditional Chinese Medicine, Beijing 100091, China \\ ${ }^{2}$ Center for Evidence-Based Chinese Medicine, Institute of Basic Research in Clinical Medicine, \\ China Academy of Chinese Medical Sciences, China \\ ${ }^{3}$ Graduate School, Chinese Academy of Traditional Chinese Medicine, Beijing 100700, China \\ ${ }^{4}$ Graduate School, Beijing University of Traditional Chinese Medicine, Beijing 100029, China
}

Correspondence should be addressed to Yunling Zhang; yunlingzhang2004@163.com and Xing Liao; okfrom2008@hotmail.com

Received 14 May 2021; Accepted 6 August 2021; Published 29 August 2021

Academic Editor: Yi Wang

Copyright (c) 2021 Min Zhan et al. This is an open access article distributed under the Creative Commons Attribution License, which permits unrestricted use, distribution, and reproduction in any medium, provided the original work is properly cited.

Background. Ginkgo biloba extract (EGb) is widely used to treat impairments in memory, cognition, activities of daily living, inflammation, edema, stroke, Alzheimer's dementia, and aging. Aim. We aimed to evaluate the safety and efficacy of EGb in treating vascular cognitive impairment (VCI). Methods. The systematic review was performed using the latest guidelines. We searched for EGb-related trials up to March 1, 2021, in four Chinese databases, three English databases, and clinical trial registry platforms. Randomized controlled trials (RCTs) were included if the study enrolled participants with VCI. Two reviewers independently extracted the data and critically appraised the study quality. Heterogeneity was quantified with $I^{2}$. Both sensitivity and subgroup analyses were used to identify the sources of heterogeneity. Publication bias was assessed with funnel plots. We used the Grading of Recommendations Assessment, Development, and Evaluation (GRADE) approach to rate the evidence quality. Outcomes included assessments using the Activities of Daily Living (ADL), Montreal Cognitive Assessment (MoCA), Mini-Mental State Examination (MMSE), Hasegawa Dementia Scale (HDS), Barthel Index (BI), Functional Activity Questionnaire (FAQ), and adverse events. Results. In this study, a total of 2019 patients in 23 RCTs were included. EGb appeared to be more effective than control conditions as assessed by the results of cognitive function evaluation, including MMSE $\left(\mathrm{MD}_{\mathrm{MMSE}, \mathrm{EGbvs} \text {.blank }}=3.04,95 \% \mathrm{CI}: 0.10-5.98 ; \mathrm{MD}_{\mathrm{MMSE}, \mathrm{EGb} \text { vs.drugs for VCI }}=2.70,95 \% \mathrm{CI}\right.$ : 1.39 4.01; $\mathrm{MD}_{\mathrm{MMSE}, \mathrm{EGb}+\text { drugs for VCI vs.blank }}=5.90,95 \% \mathrm{CI}: 4.21-7.59$; and $\mathrm{MD}_{\mathrm{MMSE}, \mathrm{EGb}+\text { drugs for VCI vs.drugs for VCI }}=3.14,95 \% \mathrm{CI}: 2.14-$ 4.15), MoCA $\left(\mathrm{MD}_{\mathrm{MoCA}, \mathrm{EGb} v \mathrm{~b} . b l a n k}=5.30,95 \%\right.$ CI: 2.15-8.46; $\quad \mathrm{MD}_{\mathrm{MoCA}, \mathrm{EG}+\text { drugs for VCI vs.blank }}=2.66,95 \%$ CI: $1.82-3.50 ;$ and $\left.\mathrm{MD}_{\mathrm{MoCA}, \mathrm{EGb}+\text { drugs for VCI vs.drugs for VCI }}=2.56, \quad 95 \% \quad \mathrm{CI}: \quad 1.85-3.27\right), \quad \mathrm{HDS} \quad\left(\mathrm{MD}_{\mathrm{HDS}, \mathrm{EGb} \text { vs.blank }}=6.50 ; \quad 95 \% \quad \mathrm{CI}: \quad 4.86-8.14 ; \quad \mathrm{M}\right.$ $\left.\mathrm{D}_{\mathrm{HDS}, \mathrm{EG}+\text { drugs for VCI vs.drugs for VCI }}=3.60, \quad 95 \% \quad \mathrm{CI}: \quad 2.50-4.70\right), \quad \mathrm{ADL} \quad\left(\mathrm{MD}_{\mathrm{ADL}, \mathrm{EGb} \text { vs.blank }}=7.20, \quad 95 \% \quad \mathrm{CI}: \quad 3.28-11.12 ; \quad \mathrm{M}\right.$ $\mathrm{D}_{\mathrm{ADL}, \mathrm{EGb}+\text { drugs for VCI vs.blank }}=10.00$, 95\% CI: 7.51-12.49; and $\mathrm{MD}_{\mathrm{ADL}, \mathrm{EGb}+\text { drugs for VCI vs.drugs for VCI }}=9.20,95 \%$ CI: 7.26-11.14), $\mathrm{BI}\left(\mathrm{MD}_{\mathrm{BI}, \mathrm{EGb}+\text { drugs for } \mathrm{VCI} \text { vs.drugs for } \mathrm{VCI}}=5.71,95 \% \mathrm{CI}: 2.99-8.43 ; \mathrm{MD}_{\mathrm{FAQ}, \mathrm{EGb} \text { vs.drugs for } \mathrm{VCI}}=-1.43,95 \% \mathrm{CI}:-2.78\right.$ to 0.08$)$, and $\mathrm{FAQ}$ $\left(\mathrm{MD}_{\mathrm{FAQ}, \mathrm{EGb}+\text { drugs for VCI vs.drugs for } \mathrm{VCI}}=-2.17,95 \% \mathrm{CI}:-4.13\right.$ to 0.21$)$. Evidence of certainty ranged from medium certainty to very low certainty. Conclusion. This meta-analysis showed that EGb may be an effective and safe treatment in improving MMSE, MOCA, $\mathrm{ADL}$, and BI for VCI patients within three months of diagnosis. However, given the quality of the included RCTs, more preregistered trials are needed that explicitly examine the efficacy of EGb. This systematic review has been registered on PROSPERO, with the registration number CRD42021232967. 


\section{Introduction}

Vascular cognitive impairment (VCI) may occur as a consequence of cardiovascular disease (CVD) and covers a broad spectrum of cognitive dysfunction, ranging from subjective cognitive decline and mild cognitive impairment to dementia $[1,2]$. There is little consistency in the overall incidence of VCI, possibly because of different settings and designs, as well as neuroimaging accessibility $[3,4]$. VCI is a clinical syndrome that occurs as a result of many different vascular pathologies $[5,6]$. As a general statement, any disease process causing cerebral ischemia or hemorrhage can cause VCI. Therefore, VCI may become the silent epidemic of the 21st century [7]. The Guidelines from the Vascular Impairment of Cognitive Classification Consensus Study (VICCCS), International Society for Vascular Behavioural and Cognitive Disorders (VASCOG), and the Diagnostic and Statistical Manual of Mental Disorders, Fifth Edition (DSM-5) have divided VCI into mild VCI and major VCI according to the severity of cognitive impairment. There are four subtypes of major VCI, including poststroke dementia (PSD), pubcortical ischemic vascular dementia (SIVaD), multi-infarct dementia (MID), and mixed dementias (MixD) $[8,9]$. The clinical features of VCI are variable, depending on the type, extent, and location of the underlying cerebrovascular pathology, and include memory problems, mental slowness, and problems with executive functions. VCI is the second most common cause of dementia, accounting for $15 \%$ of dementia cases [10], with a higher prevalence of vascular dementia ( $\mathrm{VaD}$ ) in the elderly in Asia [11]. With the rise in life expectancy over the past century, the number of people affected by dementia is likely to rise. Patients with $\mathrm{VaD}$ have a higher level of disability and higher rates of cerebrovascular diseases, congestive heart failure, hemiplegia, paraplegia, myocardial infarction, and a higher relative risk of death compared to Alzheimer disease (AD), thus increasing both the complexity and costs of management of the disease [12, 13]. VCI represents a global problem and poses a substantial economic cost to public health systems and society in Asia now and in the near future [14]. Studies have found that VCI is common and suggest it to be an important target for treatment because it may be preventable [15]. Interventions against potentially modifiable risk factors associated with VCI [16], such as controlling diabetes and hypertension and avoiding midlife obesity, among others, have been proposed as ways of reducing dementia [17].

Extracts of the leaves of the maidenhair tree, Ginkgo biloba, have long been used for treating various disorders and are one of the most widely used plant-based products. Standardized extracts are prescribed for the treatment of various disorders, including cognitive dysfunction, headache, tinnitus, vertigo, inattention, mood disturbances, cardiovascular disease [18], coronary heart disease [19, 20], and age-related macular degeneration [21]. Ginkgo biloba is mainly used in the treatment of cerebral dysfunction. The consensus of the Asian Clinical Expert Group on Neurocognitive Disorders in 2019 recommended EGb as an important part of the clinical treatment of neurodegenerative diseases, such as $\mathrm{AD}$, which has received widespread attention $[22,23]$. The active components of Ginkgo biloba consist of flavonoids, terpenoids, ginkgolides, and bilobalide. Ginkgo biloba has been demonstrated to have antioxidative activity and has been shown to restore impaired mitochondrial function, thereby improving the neuronal energy supply, as well as improving compromised hippocampal neurogenesis and neuroplasticity [24], inhibiting the aggregation and toxicity of the amyloid $\beta$-peptide [25], decreasing blood viscosity, enhancing microperfusion [26], and increasing dopamine levels in the rat prefrontal cortex thus enhancing working memory and executive control [27]. Current studies have shown that EGb can influence the PI3K/Akt, CREB, and RSK1/GSK-3 $\beta$ signaling pathways to play a neuroprotective role [28]. Two well-defined extracts, EGb 761 and Kaveri (LI 1370), are produced from the ground leaves. In Germany, EGb 761 is one of the top five prescription medicines, while it is marketed as a food supplement and available without prescription in the UK, Canada, and the USA [28]. Ginkgo biloba has been the subject of many research reports and has been investigated in numerous clinical trials. Many systematic reviews covering different aspects of Ginkgo biloba have been published [21, 28, 29].

Despite the number of clinical trials conducted to assess its potential properties $[30,31]$ and the publication of several reviews documenting its efficacy in the prevention of cognitive decline and for treating cognitive impairment and dementia [32], there is still no compelling evidence on the efficacy of EGb for VCI. Therefore, we conducted a systematic review to evaluate the efficacy and safety of EGb for VCI.

\section{Materials and Methods}

This meta-analysis is reported in accordance with the Preferred Reporting Items for Systematic Reviews and MetaAnalyses (PRISMA) statement [33], displayed in Appendix 1.

2.1. Data Sources and Searches. Relevant studies were identified by searching seven databases and two trial registration platforms from their inception to March 1, 2021. The databases included the Chinese Biological Medical Literature Database, Chinese Wanfang data, Chinese VIP information, Chinese National Knowledge Infrastructure, PubMed, EMBASE, and the Cochrane library. The trial registration platforms were the China Clinical Trial Registration Center (ChiCTR) and ClinicalTrials.gov. We also checked the reference lists of all retrieved articles and relevant review articles to identify additional studies. We only paid attention to studies published in English and Chinese. Two individual reviewers (MZ and $\mathrm{ZXZ}$ ) screened the titles and abstracts to select relevant studies, and duplicate studies were removed after screening each article's abstract and title. Subsequently, the eligibility criteria were used to review full-text manuscripts for available data. Any disagreements were settled via a consensus with a third researcher (XL). The detailed search strategies are shown in Appendix 2.

2.2. Study Selection. This systematic review employed the PICOS strategy, an abbreviation of patient, intervention, comparison, and outcome which was used for all steps in 
the current systematic review. Firstly, randomized controlled trials (RCTs) that examined the efficacy of EGb for VCI were included. Secondly, we used VCI as the umbrella term encompassing vascular dementia and other cognitive syndromes with a presumed vascular basis (including mild VCI and all subdivisions of major VCI). Thirdly, we included trials with the intervention of EGb (tablets) alone or combined with a drug for VCI (hereafter referred to as DV and mainly including drugs to promote microcirculation and improve cognition, such as donepezil, nimodipine, huperzine, oxiracetam, piracetam, and butylphthalide, among others). There are three common forms of oral Ginkgo leaf products, namely, tablets, capsules, and soft capsules. It has been found in clinical practice that patients prefer tablets than the other two forms. Fourthly, the control therapy could be any kind of DV, blank, or placebo. Normally, patients would be prescribed some basic supporting treatments, including symptomatic treatment for hyperglycemia, hyperlipidemia, and hypertension. The blank or placebo would then be added to the basic supporting treatments. Fifthly, the outcomes covered measurements of cognitive function, including the MiniMental State Examination (MMSE), Montreal Cognitive Assessment (MoCA), and Hasegawa Dementia Scale (HDS), as well as the evaluation of daily activities, including the Activities of Daily Living (ADL), Barthel Index (BI), and Functional Activity Questionnaire (FAQ) assessments, and safety was assessed by the occurrence of adverse reactions/events. We excluded studies if they included any of the following: (1) no available full text; (2) non-RCTs (i.e., editorials, commentaries, and letters to the editor); (3) studies with faulty data; and (4) duplicate studies.

2.3. Data Extraction and Quality Assessment. All related records from databases and platforms were imported into the literature management software NoteExpress 3.2.0. The eligible studies were independently screened and selected by two reviewers (MZ and ZXZ). Then, the key information was extracted from the included studies using standardized data extraction forms, including information on the authors and study design, participant characteristics, details of the intervention and control groups, and the outcomes. The risk of bias of the included trials was evaluated by two reviewers (MZ and ZXZ) according to the Cochrane risk of bias assessment tool (Cochrane Reviewers Handbook version 6.1) [34]. Seven items were evaluated, including (1) random sequence generation, (2) allocation hiding, (3) blind setting (researchers, subjects), (4) blind evaluation of study outcomes, (5) data integrity of outcomes, (6) selective reporting of research results, and (7) Other sources of bias (such as potential bias related to special research design, baseline imbalance, and suspected fraud). Each item was evaluated as being "low risk," "unclear," or "high risk" and was independently completed by two evaluators (MZ and ZXZ). Disagreements during the study screening, data extraction, and quality appraising were resolved by consulting a third reviewer (XL).

2.4. Data Synthesis and Analysis. The GRADE (the Grading of Recommendations Assessment, Development, and Evaluation) system [35] was used to rate the quality of a body of evidence across outcomes. GRADE has four levels of evidence, also known as certainty in evidence or quality of evidence: very low, low, moderate, and high. We assessed the five aspects for each outcome (risk of bias, inconsistency, imprecision, indirectness, and publication bias) to evaluate the quality of the body of evidence as it related to the studies that contributed data to the meta-analyses. We created a "Summary of findings" table to summarize the effects of interventions on key outcomes, including MMSE, MoCA, HDS, ADL, BI, FAQ, and serious adverse events. We used GRADEpro GDT [36] to create the "Summary of findings." Explanations for downgrading the quality of the evidence were listed in the footnotes.

Data analysis was performed by Review Manager 5.3 software [37] provided by the Cochrane Collaboration. We calculated the weighted mean difference (WMD) with $95 \%$ confidence interval (CI) for continuous data, and the risk ratio (RR) with $95 \% \mathrm{CI}$ was computed for the dichotomous data. For continuous data, if the outcome was measured on different assessment scales (such as pain), we calculated the standardized mean differences (SMDs) with 95\% CIs. Before we performed the meta-analysis, the clinical heterogeneity and methodological heterogeneity were assessed. If there was no clinical or methodological heterogeneity, the chisquare test with a significance level at $P<0.1$ and the $I^{2}$ statistic were used to quantify possible heterogeneity. If $P \geq$ 0.10 and $I^{2}<50 \%$, there was no statistical heterogeneity between the studies in the meta-analysis and the fixed effects model was used for analysis. If $P<0.10$ and $I^{2}>50 \%$, this was considered to represent substantial heterogeneity between studies in the meta-analysis, and the random effects model was used to pool the data. Sensitivity or subgroup analyses were performed to determine the reasons for heterogeneity and whether the random effects model could be used for analysis or not. Descriptive analysis was used if the clinical heterogeneity was too large, or there were insufficient reports to perform a meta-analysis. All analyses were two-tailed, with alpha set at 0.05 , except for heterogeneity. Publication bias was assessed using funnel plots for more than 10 studies with a particular outcome.

\section{Results}

3.1. Literature Search and Trial Selection. The original search of the above nine databases yielded 57,821 electronic records, including 6462 records in English and 51,359 records in Chinese. After screening the titles and abstracts, the full text of 3288 articles was reviewed. Ultimately, 23 eligible studies were selected for the present review [38-60] and were included in the qualitative and quantitative synthesis. The screening process is summarized in a flow diagram shown in Figure 1. A self-evaluation according to the PRISMA checklist is shown in Appendix 1.

3.2. Description of the Included Trials. In this study, 23 RCTs on the use of EGb in the treatment of VCI were included. All trials were performed in mainland China and published in Chinese. A total of 2019 patients were included, including 1012 patients in the experimental group and 1007 patients 

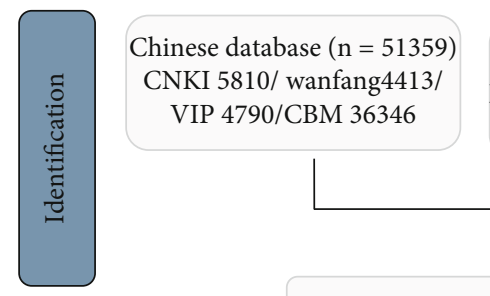

English database $(n=6462)$ pubmed 2435/cochrane 836/ Embase 3191
$\operatorname{ChiCTR}(\mathrm{n}=0)$

ClinicalTrials.govn $=0$
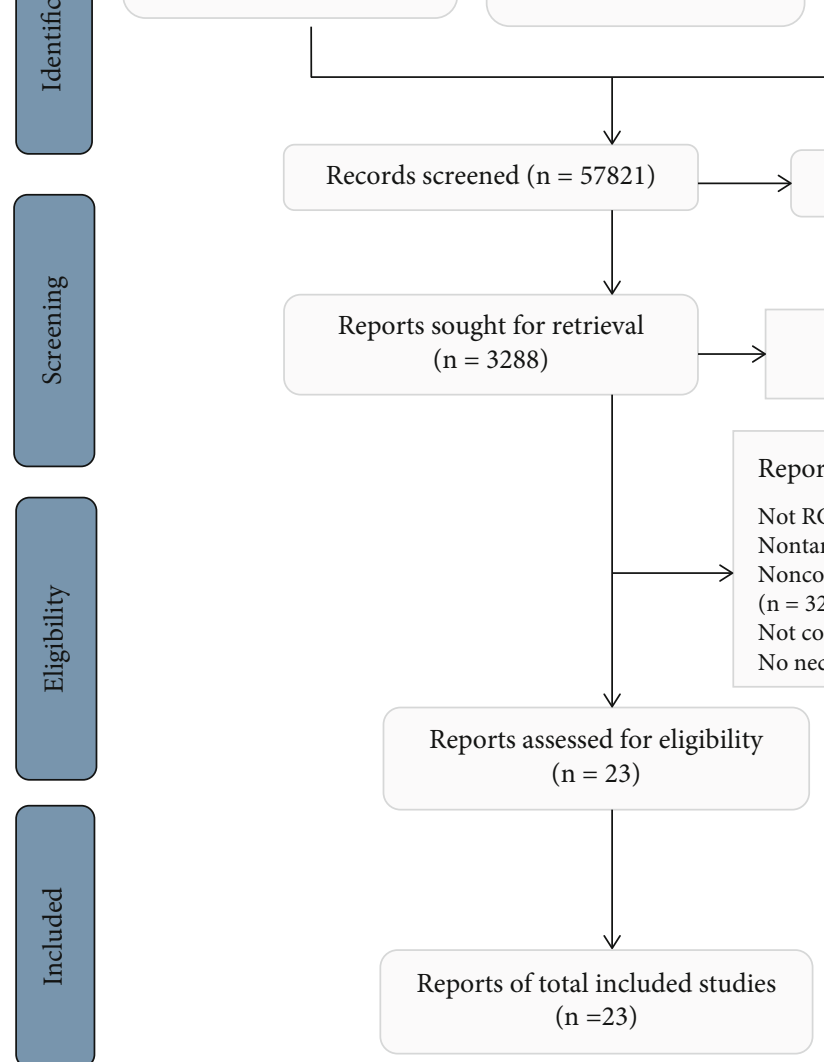

FIGURE 1: Flow chart of the study selection process.

in the control group. The patients' ages ranged from 49 to 83 years. There were no significant differences in sex, age, course of disease, or condition of the subjects between the study groups, with comparable baselines. Among the 23 RCTs, four assessed poststroke cognitive impairment (PSCI) $[44,50,51,56]$; three studied vascular cognitive impairment, no dementia (VCIND) $[47,53,59]$; one did not mention sepcial subtype [42]; the remaining 15 reported on vascular dementia (VD) $[38-41,43,45,46,48,49,52,54,55,57$, $58,60]$. Eleven studies [39, 42-45, 47, 48, 52, 53, 55, 56] used the diagnostic criteria of the Neurology Society of Chinese Medical Association, seven studies [38, 40, 41, 46, 49, 54, 57] used the American Psychiatric Association criteria, and the remaining five studies [50, 51, 58-60] were unclear. Sixteen RCTs [38, 40-47, 49-54, 60] used EGb in combination with DV (eight trials [42-45, 50-52, 60] with donepezil, three trials $[38,47,53]$ with nimodipine, five trials [40, 41, $46,49,54]$ with huperzine, oxiracetam, piracetam, butylphthalide, ergoloid, and XueSaiTong) as the treatment group versus DV as the control group. Two RCTs [48, 57] used EGb as the monotherapy in the treatment group versus DV alone in the control group. Three RCTs $[39,55,59]$ used $\mathrm{EGb}$ as the monotherapy in the treatment group versus a blank group. The duration of studies lasted from two to six months. As the outcome measurements, sixteen studies
[38-40, 43, 44, 46, 48-52, 54-57, 60] used MMSE, nine studies $[39,42,45-47,50,53,58,59]$ used MoCA, six studies $[38-41,45,56]$ used ADL, three studies [44, 51, 55] used HDS, four studies $[43,46,54,60]$ used BI, and three studies $[48,49,57]$ used FAQ. The total clinical efficacy rate was observed in 11 studies [41, 43-45, 48-50, 54, 55, 57, 60]. Adverse effects were reported in 15 studies [38-40, 43-45, $48-52,54,56,57,60]$. The characteristics of the 23 trials are summarized in Table 1.

3.3. Risk of Bias Assessment of the Included Studies. All 23 trials claimed randomization, however, none of them described the allocation of concealment methods, and none used placebo controls or registered their protocols. The method of random sequence generation was described in eight trials as a random number table $[41-44,47,50$, 53, 54]; others did not report specific methods. Only one trial used a single-blind method for patients [42]; one trial used a double-blind method for both patients and researchers [46]. Adverse events were reporeted in 15 trials; only one trial reported dropout [38]. The selective reporting assessments of all RCTs were defined as "low" for their clear inclusion and exclusion criteria. The results of the risk of bias assessment of the included studies are shown in Figure 2. 


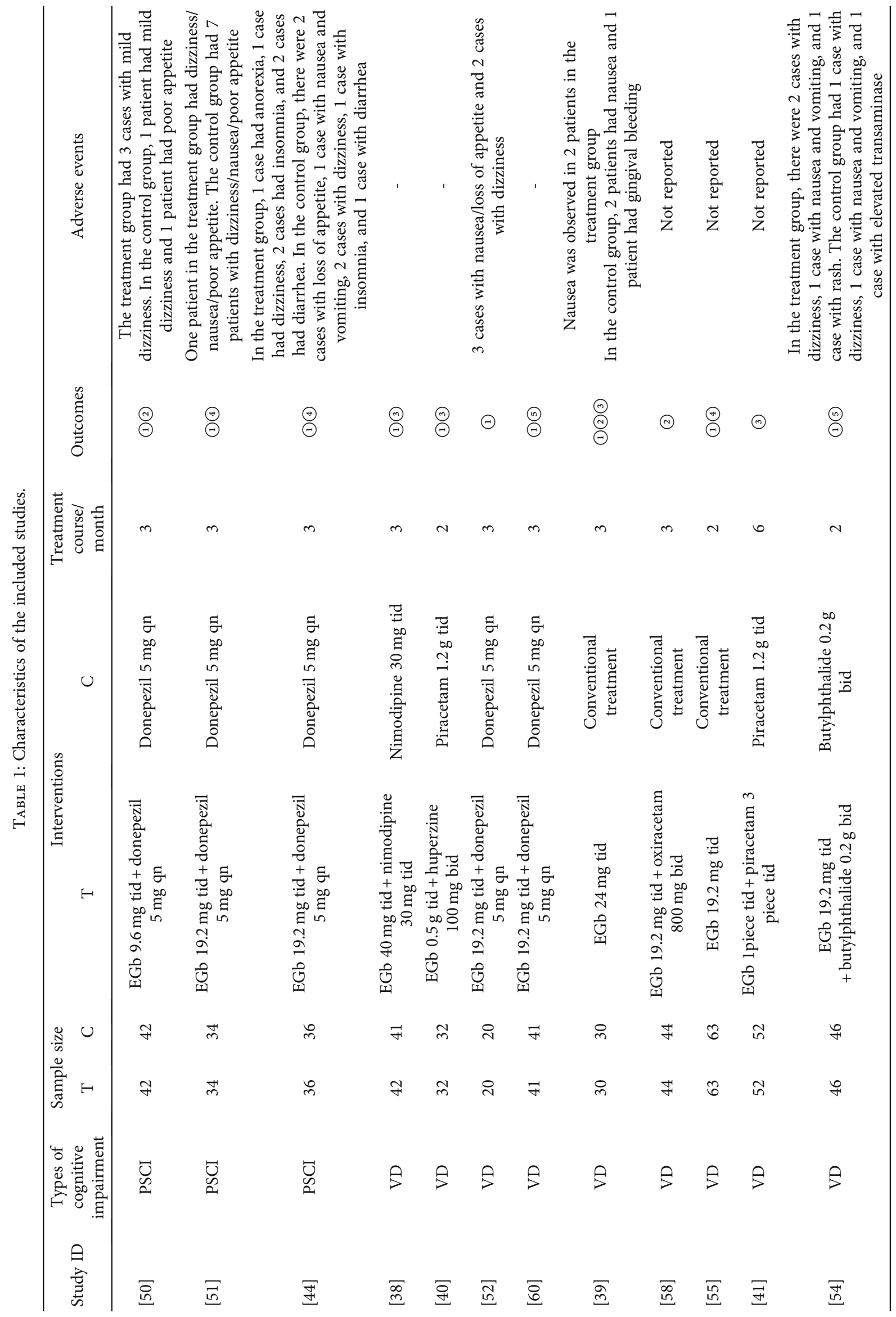




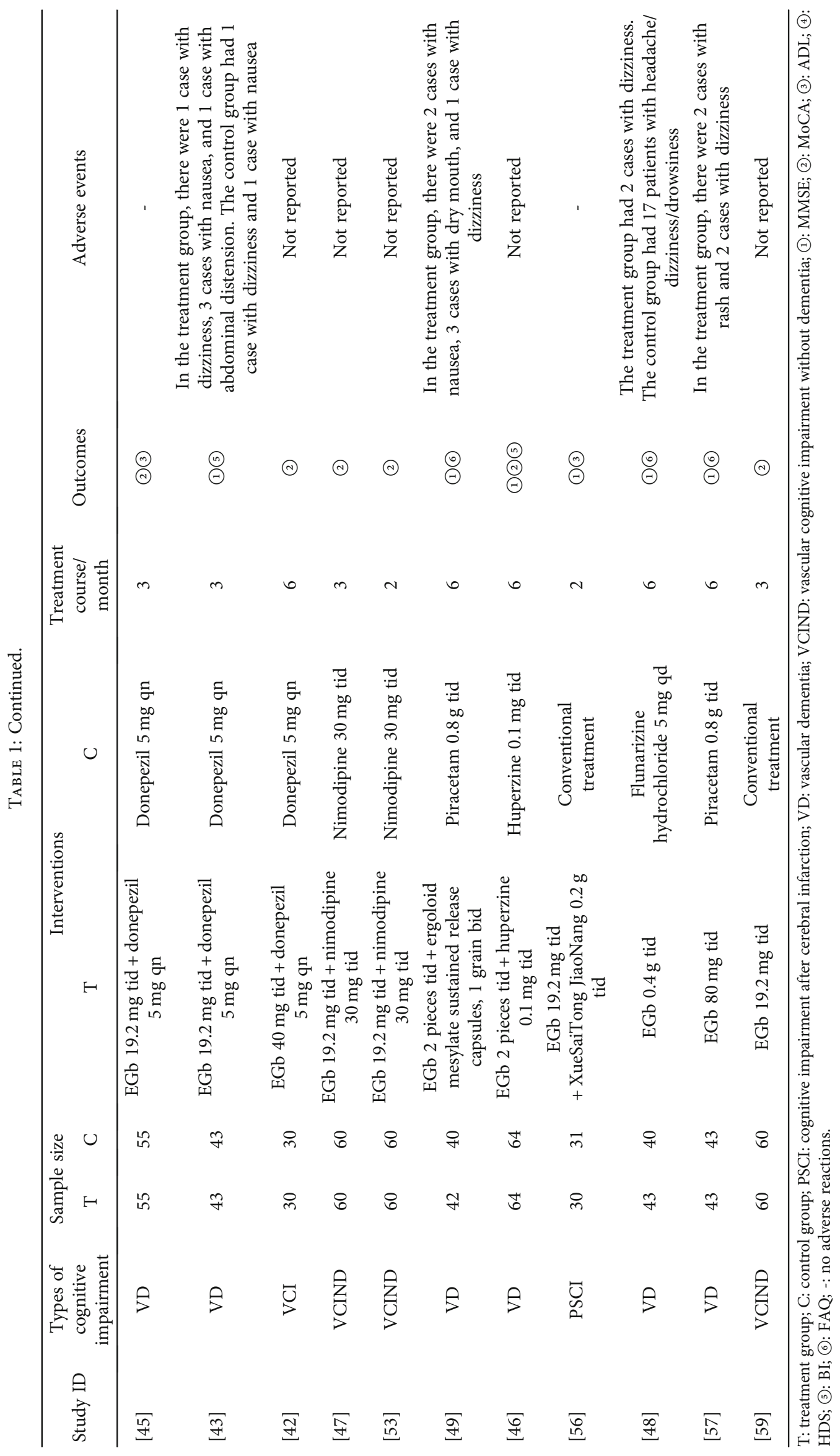




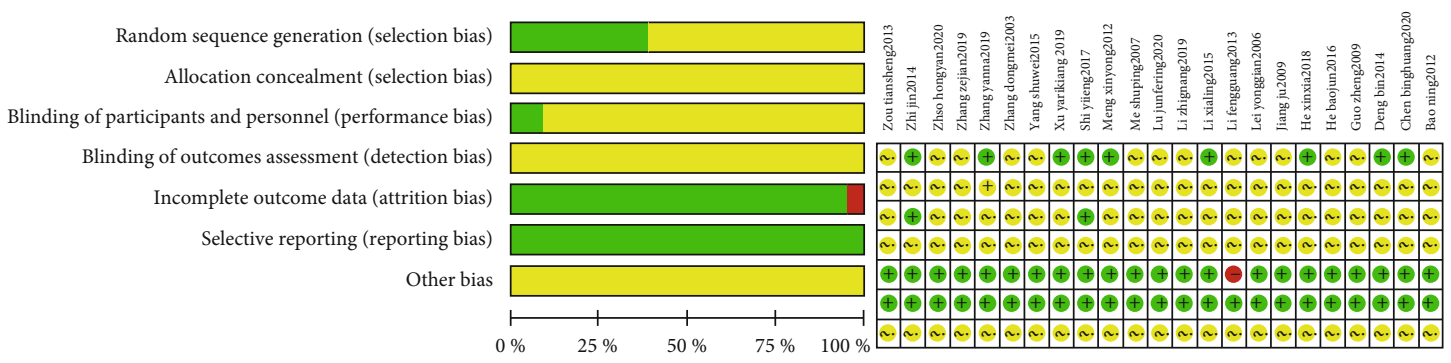

Low risk of bias

Unclear risk of bias

High risk of bias

Figure 2: Methodological quality graph: review authors' judgments about each methodological quality item presented as percentages across all included studies and each item for each included study.

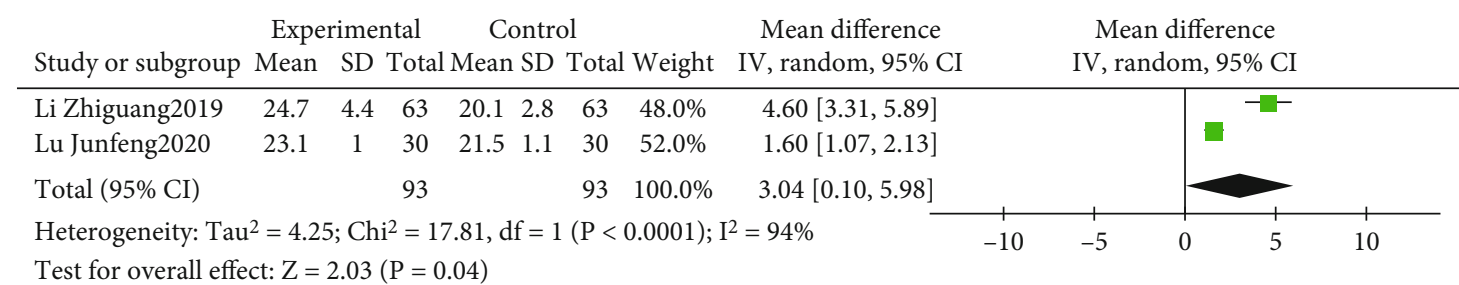

Favours [control] Favours [experimental]

FIGURE 3: Forest plot of comparison: EGb versus blank group on MMSE levels.

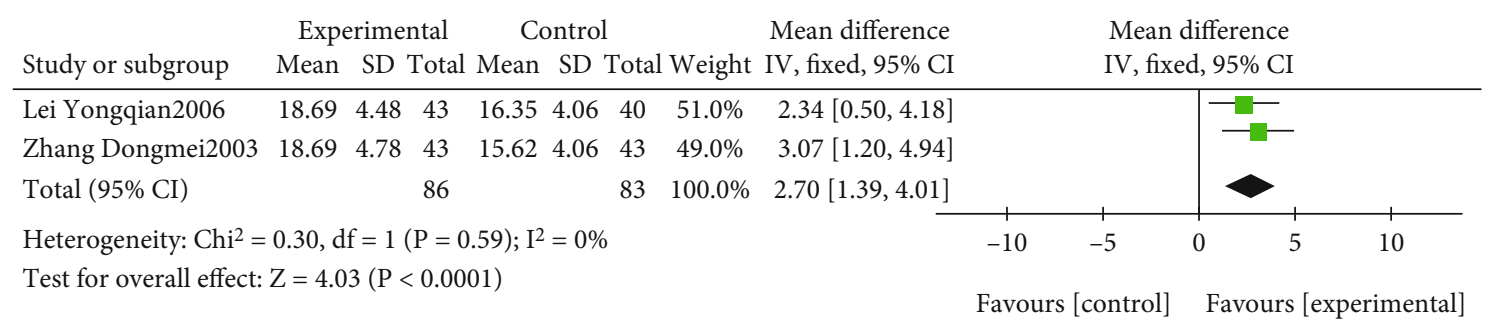

Figure 4: Forest plot of comparison: EGb versus drugs for VCI on MMSE levels.

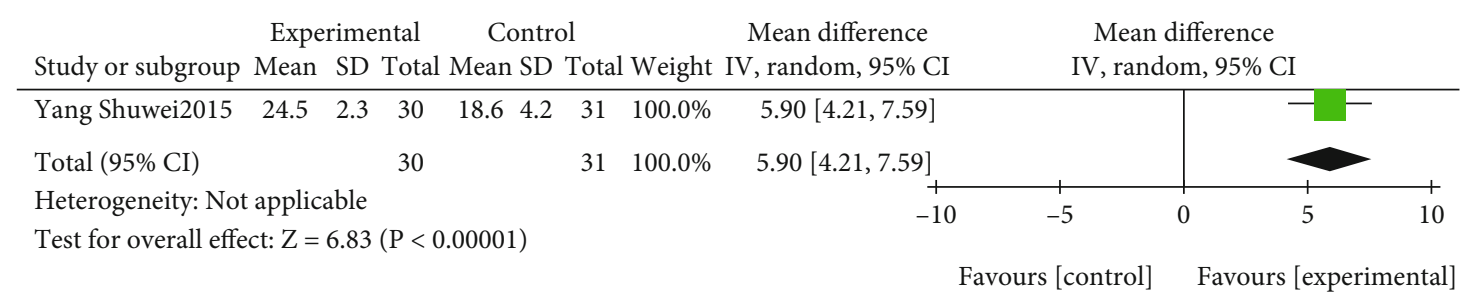

FIGURE 5: Forest plot of comparison: EGb combined with drugs for VCI versus blank group on MMSE levels.

\subsection{Meta-Analysis}

3.4.1. Analysis of MMSE. The effect of EGb compared to the blank on MMSE is summarized in Figure 3. We used random effects models for pooling the effect estimates in two studies ( $n=186$ patients) $[39,55]$. There was a significant difference in favor of EGb for improving MMSE (MD: 3.04; 95\% CI: $0.10-5.98 ; P=0.04)$. However, the heterogeneity was substantial $\left(I^{2}=94 \%\right)$.
The effect of EGb compared to DV on MMSE is outlined in Figure 4. We employed fixed effects models for pooling the effect estimates in two studies ( $n=169$ patients) [48, 57]. There was a significant difference in favor of EGb for improving MMSE (MD: 2.70; 95\% CI: 1.39-4.01; $P<$ $\left.0.0001 ; I^{2}=0 \%\right)$.

The effects of EGb combined with DV compared to the blank on MMSE are summarized in Figure 5. Only one study ( $n=61$ patients) [56] investigated the effect of EGb in 


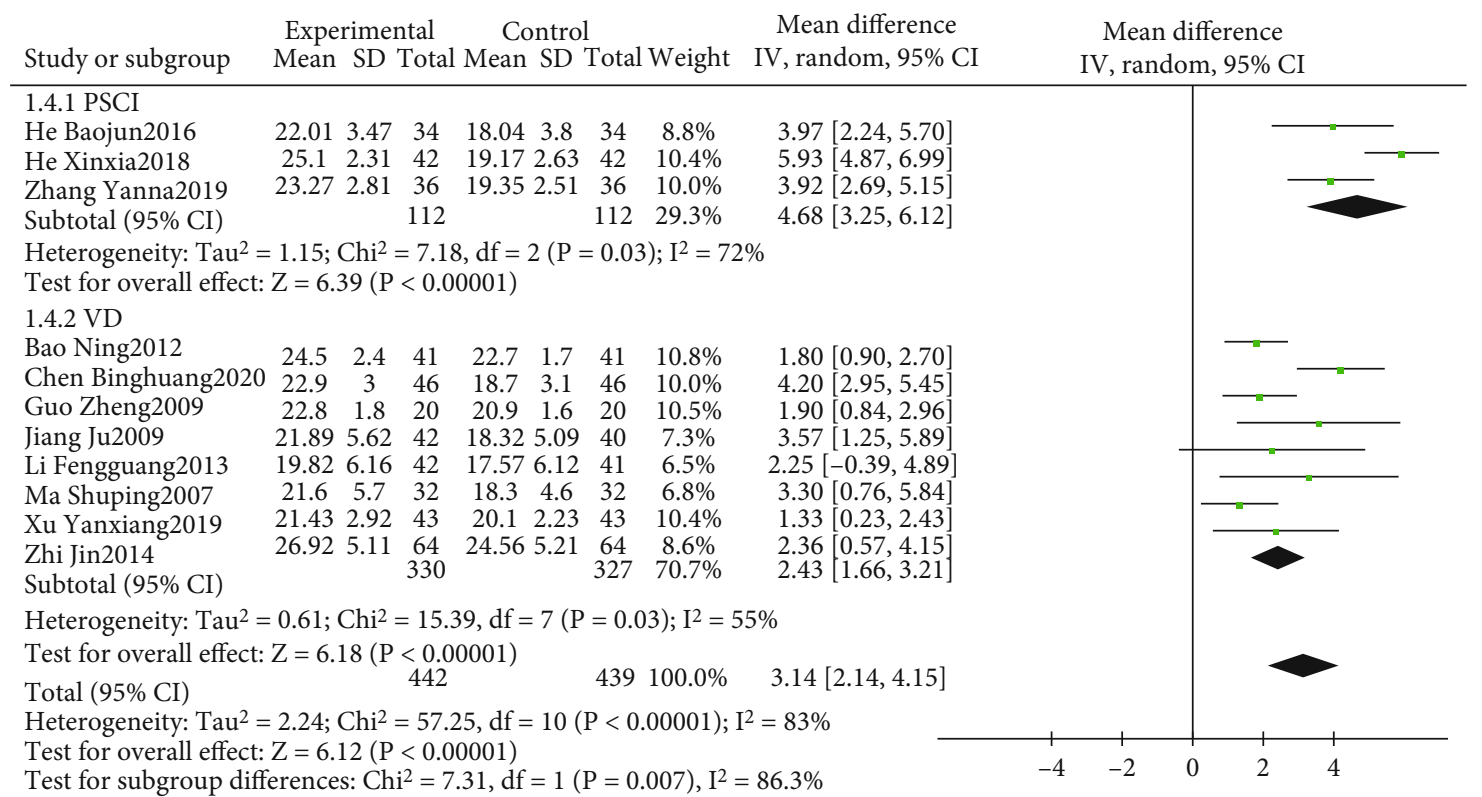

Favours [control] Favours [experimental]

FIGURE 6: Forest plot of comparison: EGb combined with drugs for VCI versus drugs for VCI on MMSE levels (different types of cognitive impairment).

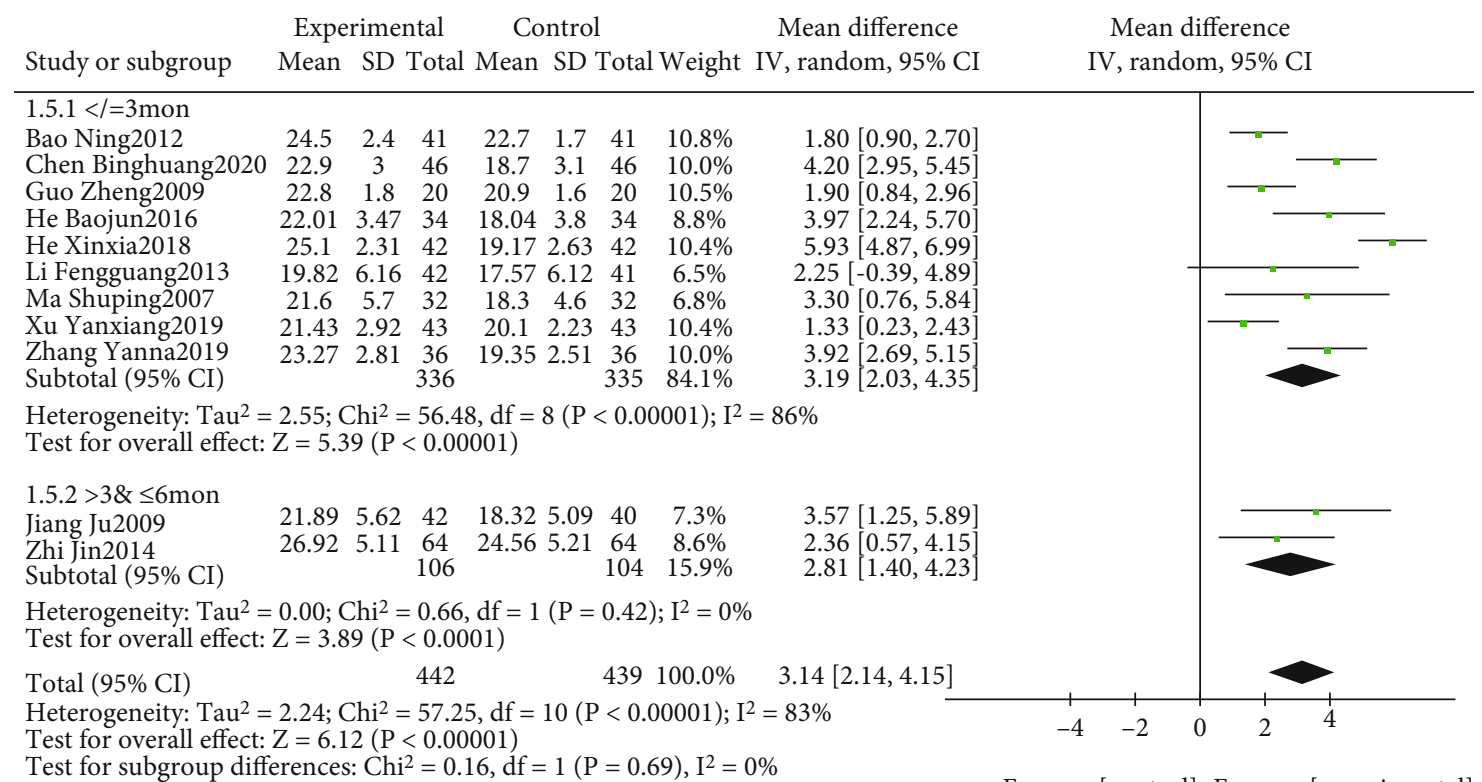

Favours [control] Favours [experimental]

FIGURE 7: Forest plot of comparison: EGb combined with drugs for VCI versus drugs for VCI on MMSE levels (different treatment courses).

Experimental Control Mean difference Mean difference

Study or subgroup Mean SD Total Mean SD Total Weight IV, random, 95\% CI IV, random, 95\% CI

FIGURE 8: Forest plot of comparison: EGb versus blank group on MoCA levels. 


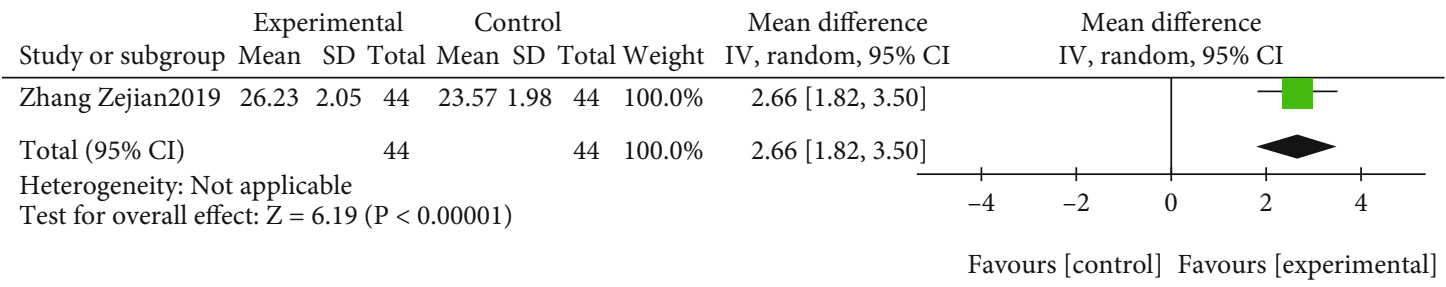

FIGURE 9: Forest plot of comparison: EGb combined with drugs for VCI versus blank group on MoCA levels.

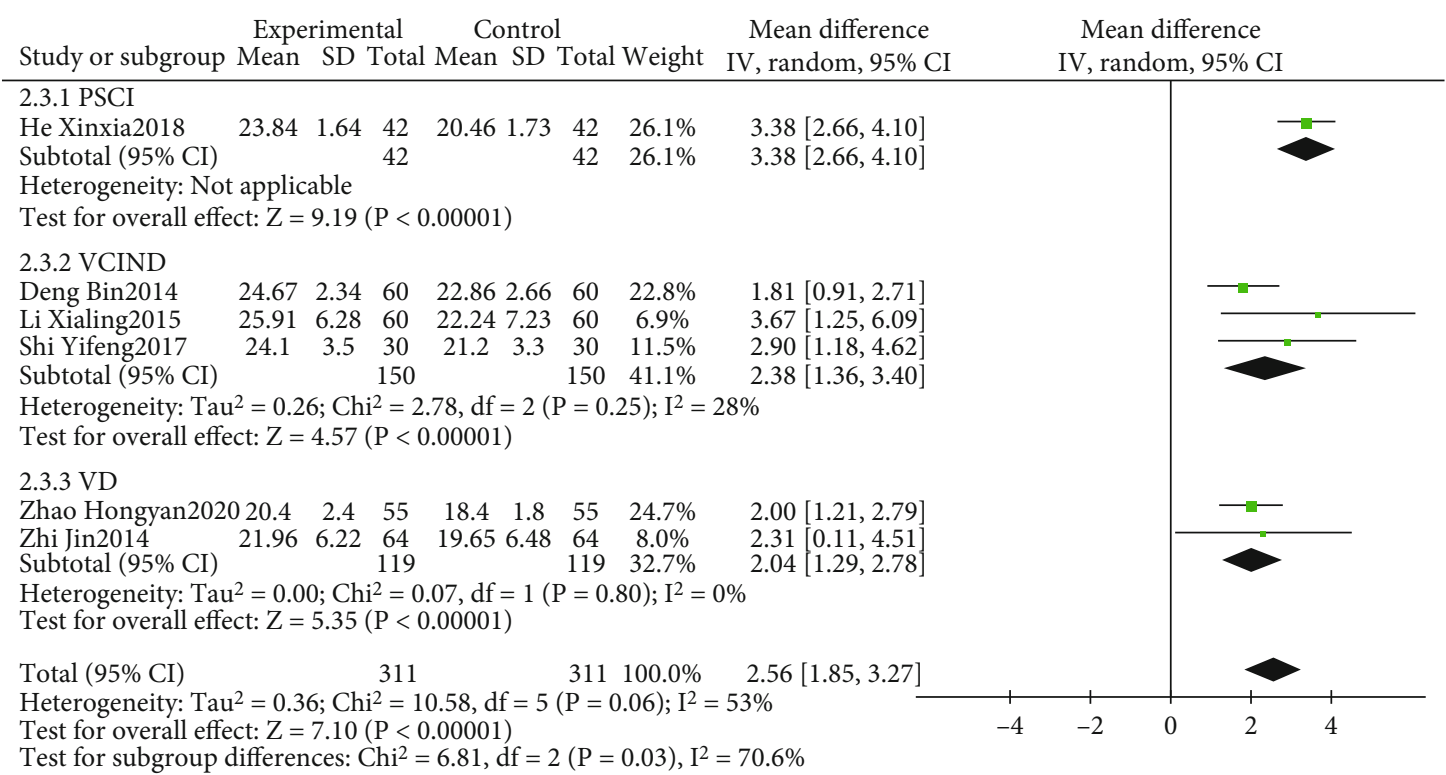

Favours [control] Favours [experimental]

FIGURE 10: Forest plot of comparison: EGb combined with drugs for VCI versus drugs for VCI on MoCA levels (different types of cognitive impairment).

conjunction with DV. A significant difference was reported in favor of EGb in conjunction with DV to improve MMSE (MD: 5.90; 95\% CI: 4.21-7.59; $P<0.00001$ ).

The effect of EGb combined with DV compared to DV alone on MMSE is summarized in Figures 6 and 7. We used random effects models for pooling the effect estimates from 11 trials ( $n=881$ patients) [38, 40, 43, 44, 46, 49-52, 54, 60]. There was a significant difference in favor of EGb with DV for improving MMSE (MD: 3.14; 95\% CI: 2.14-4.15; $P<$ $0.00001)$. However, the heterogeneity was substantial $\left(I^{2}=83 \%\right)$. Subgroup analysis was conducted according to the different types of cognitive impairment and different courses of intervention. There was a significant difference in favor of EGb with DV for improving MMSE in three studies concerning PSCI (MD: 4.68; 95\% CI: 3.25-6.12; $P<$ $\left.0.00001 ; I^{2}=72 \%\right)$ and in eight studies concerning VD (MD: 2.43 ; 95\% CI: $1.66-3.21 ; P<0.00001 ; I^{2}=55 \%$ ). There was a significant difference in favor of EGb with DV for improving MMSE in nine studies with three-month treatment courses (MD: 3.19; 95\% CI: 2.03-4.35; $P<0.00001$; $\left.I^{2}=86 \%\right)$ and in two studies with six months of treatment (MD: $2.81 ; 95 \%$ CI: $1.40-4.23 ; P<0.0001 ; I^{2}=0 \%$ ).

3.4.2. Analysis of MoCA. The effect of EGb compared to the blank on MoCA is summarized in Figure 8. We used ran- dom effects models for pooling the effect estimates from two trials ( $n=180$ patients) $[39,59]$. There was a significant difference in favor of EGb for improving MoCA (MD: 5.30; 95\% CI: 2.15-8.46; $P=0.001)$. However, the heterogeneity was substantial $\left(I^{2}=95 \%\right)$.

The effect of EGb combined with DV compared to the blank on MoCA is summarized in Figure 9. Only one study ( $n=88$ patients) [58] investigated the effect of EGb in conjunction with DV. A significant difference was reported in favor of EGb in conjunction with DV to improve MoCA (MD: 2.66; 95\% CI; 1.82-3.50; $P<0.00001$ ).

The effect of EGb combined with DV compared to DV alone on MoCA is summarized in Figures 10 and 11. We used random effects models for pooling the effect estimates from six trials ( $n=622$ patients) [42, 45-47, 50, 53]. There was a significant difference in favor of EGb with DV for improving MoCA levels (MD: 2.56; 95\% CI: 1.85-3.27; $P=$ $0.006 ; I^{2}=53 \%$ ). Subgroup analysis was conducted according to the different types of cognitive impairment and different courses of intervention. There was a significant difference in favor of EGb with DV for improving MoCA in one study concerning PSCI (MD: 3.38; 95\% CI: 2.66-4.10; $P<0.00001)$ and in two studies concerning VD (MD: 2.04; 95\% CI: 1.29-2.78; $\left.P<0.00001 ; I^{2}=0 \%\right)$. There was a significant difference in favor of EGb with DV for improving 


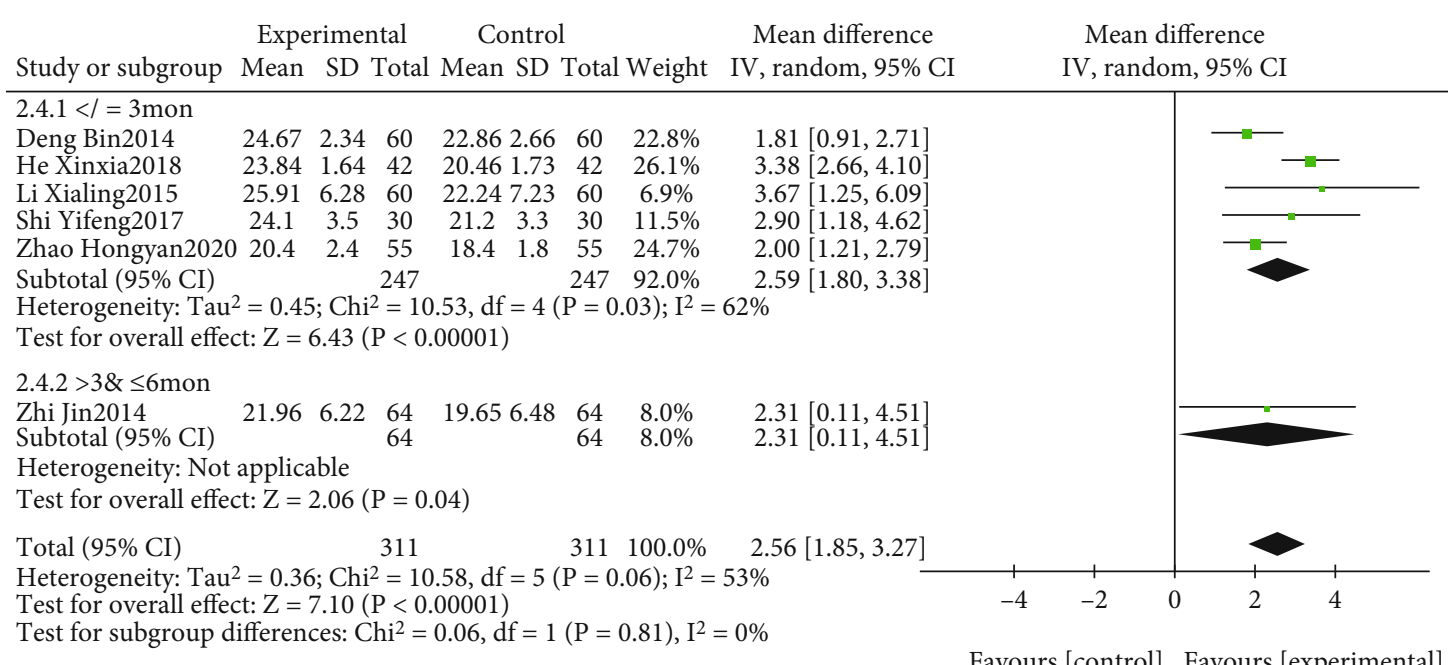

FIGURE 11: Forest plot of comparison: EGb combined with drugs for VCI versus drug treatment only for VCI on MoCA levels (different courses of treatment).

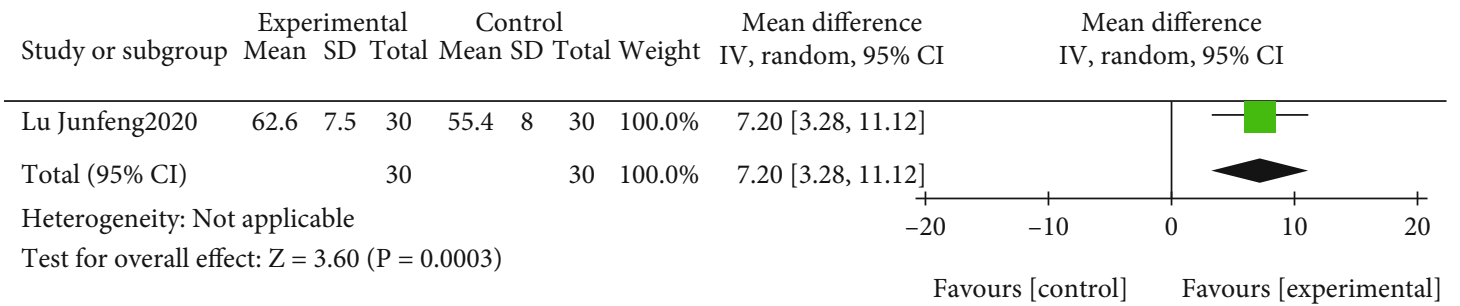

FIGURE 12: Forest plot of comparison: EGb versus blank group on ADL levels.

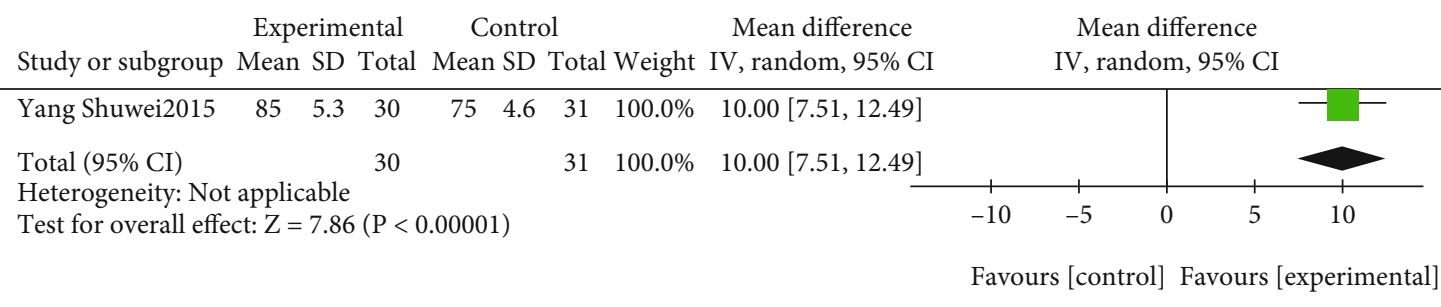

FIGURE 13: Forest plot of comparison: EGb combined with drugs for VCI versus blank group on ADL levels.

MoCA in five studies with three-month treatment courses (MD: 2.59; 95\% CI: $1.80-3.38 ; P<0.00001 ; I^{2}=62 \%$ ) and in one study with six months of treatment (MD: $2.31 ; 95 \%$ CI: $0.11-4.51 ; P=0.04)$.

3.4.3. Analysis of $A D L$. The effect of EGb compared to the blank on ADL is summarized in Figure 12. Only one trial ( $n=60$ patients) [39] reported a significant difference in favor of EGb for improving ADL (MD: 7.20; 95\% CI: 3.2811.12; $P=0.0003)$.

The effect of EGb combined with DV compared to the blank on ADL is summarized in Figure 13. Only one study ( $n=61$ patients) [56] investigated the effect of EGb in conjunction with DV. A significant difference was reported in favor of EGb in conjunction with $\mathrm{DV}$ to improve ADL (MD: 7.20; 95\% CI: 3.28-11.12; $P=0.0003$ ).

The effect of EGb combined with DV compared to DV on ADL is summarized in Figure 14. We used fixed effects models for pooling the effect estimates from four trials ( $n=361$ patients) $[38,40,41,45]$. There was no significant difference between the two groups (MD: 9.20; 95\% CI: 7.26-11.14; $\left.P=0.44 ; I^{2}=0 \%\right)$. Subgroup analysis was conducted according to different courses of intervention. There was a significant difference in favor of EGb with DV for improving ADL in five studies with three-month treatment courses (MD: $8.00,95 \%$ CI: 5.59-10.41; $P<0.00001 ; I^{2}=0 \%$ ) and in one study with six months of treatment (MD: 11.41; 95\% CI: 8.14-14.68; $P<0.00001)$.

3.4.4. Analysis of HDS. The effect of EGb compared to the blank on HDS is summarized in Figure 15. Only one trial ( $n=126$ patients) [55] reported that a significant difference in favor of EGb for improving HDS (MD: 6.50; 95\% CI: 4.86-8.14; $P<0.00001)$.

The effect of EGb combined with DV compared to DV on HDS is summarized in Figure 16. We used random 


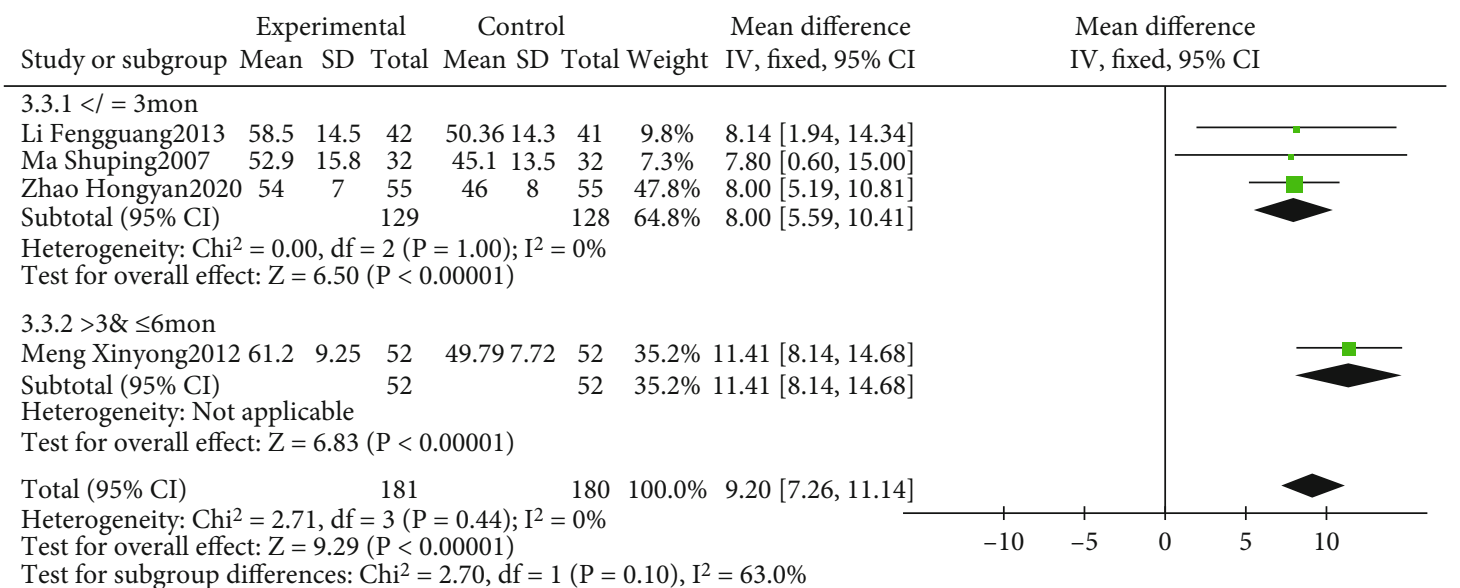

Favours [control] Favours [experimental]

FIGURE 14: Forest plot of comparison: EGb combined with drugs for VCI versus drug treatment only for VCI on ADL levels (different treatments).

\begin{tabular}{|c|c|c|c|c|c|c|c|c|c|c|c|}
\hline \multirow{2}{*}{$\begin{array}{l}\text { Study or Subgroup } \\
\text { Li Zhiguang2019 }\end{array}$} & \multicolumn{3}{|c|}{ Experimental } & \multicolumn{2}{|c|}{ Control } & Weight & $\begin{array}{c}\text { Mean difference } \\
\text { IV, random, 95\% CI }\end{array}$ & \multicolumn{3}{|c|}{$\begin{array}{l}\text { Mean difference } \\
\text { IV, random, 95\% CI }\end{array}$} & \\
\hline & 24.8 & 5.8 & 63 & $18.3 \quad 3.2$ & 63 & $100.0 \%$ & $6.50[4.86,8.14]$ & & & & \\
\hline Total $(95 \%$ CI $)$ & & & 63 & & 63 & $100.0 \%$ & $6.50[4.86,8.14]$ & & & 4 & \\
\hline $\begin{array}{l}\text { Heterogeneity: Not } \\
\text { Test for overall effe }\end{array}$ & $\begin{array}{l}\text { applic } \\
\text { ct: } Z=\end{array}$ & & & $.00001)$ & & & -1 & -5 & 0 & 5 & 10 \\
\hline
\end{tabular}

FIGURE 15: Forest plot of comparison: EGb versus blank group on HDS levels.

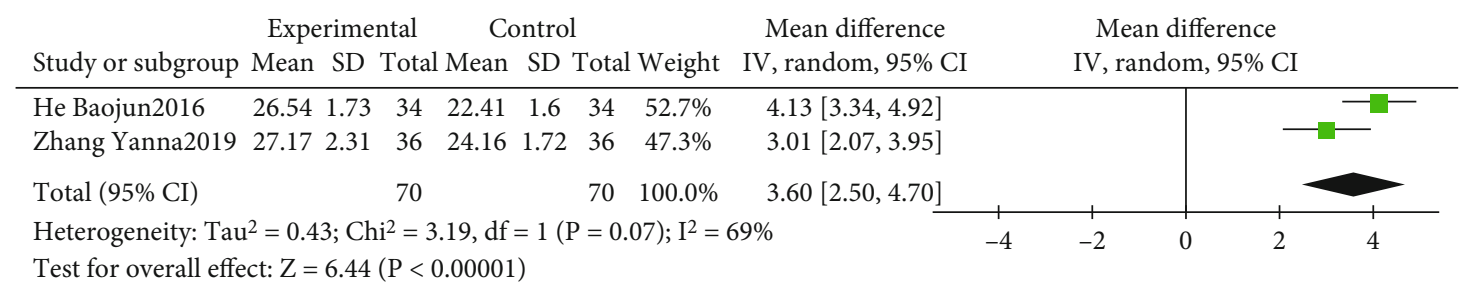

Favours [control] Favours [experimental]

FIgURE 16: Forest plot of comparison: EGb combined with drugs for VCI versus drug treatment only for VCI on HDS levels.

effects models for pooling the effect estimates from two trials ( $n=140$ patients) $[44,51]$. There was a significant difference between the two groups (MD: 3.60; 95\% CI: 2.50-4.70; $P<$ $0.00001 ; I^{2}=69 \%$ ).

3.4.5. Analysis of BI. The effect of EGb combined with DV compared to DV alone on BI is summarized in Figure 17. We used random effects models for pooling the effect estimates from four trials ( $n=388$ patients) [43, 46, 54, 60]. There was a significant difference between the two groups (MD: 5.71; 95\% CI: 2.99-8.43; $P=0.0002 ; I^{2}=85 \%$ ). Subgroup analysis was conducted according to different intervention courses. There was a significant difference in favor of EGb with DV for improving BI in three studies with three-month treatment courses (MD: 6.78; 95\% CI: 3.649.91; $\left.P<0.0001 ; I^{2}=84 \%\right)$ and in one study with six months of treatment (MD: 2.59 ; $95 \%$ CI: $0.52-4.66 ; P=0.01$ ).
3.4.6. Analysis of FAQ. The effect of EGb compared to DV alone on FAQ is summarized in Figure 18. We used fixed effects models for pooling the effect estimates from two trials ( $n=169$ patients) $[48,57]$. There was a significant difference in favor of EGb for improving FAQ (MD: -1.43; 95\% CI: -2.78 to $0.08 ; P=0.04 ; I^{2}=0 \%$ ).

The effect of EGb combined with DV compared to DV alone on FAQ is summarized in Figure 19. Only one study ( $n=82$ patients) [49] investigated the effect of EGb in conjunction with DV. A significant difference was reported in favor of EGb in conjunction with $\mathrm{DV}$ to improve $\mathrm{FAQ}$ (MD: $-2.17 ; 95 \%$ CI: -4.13 to $0.21 ; P=0.03$ ).

3.5. Adverse Events. Adverse effects were reported in 15 studies [38-40, 43-45, 48-52, 54, 56, 57, 60] of the 23 included studies, but were not mentioned in the remaining eight studies $[41,42,46,47,53,55,58,59]$. Five out of the 15 studies 


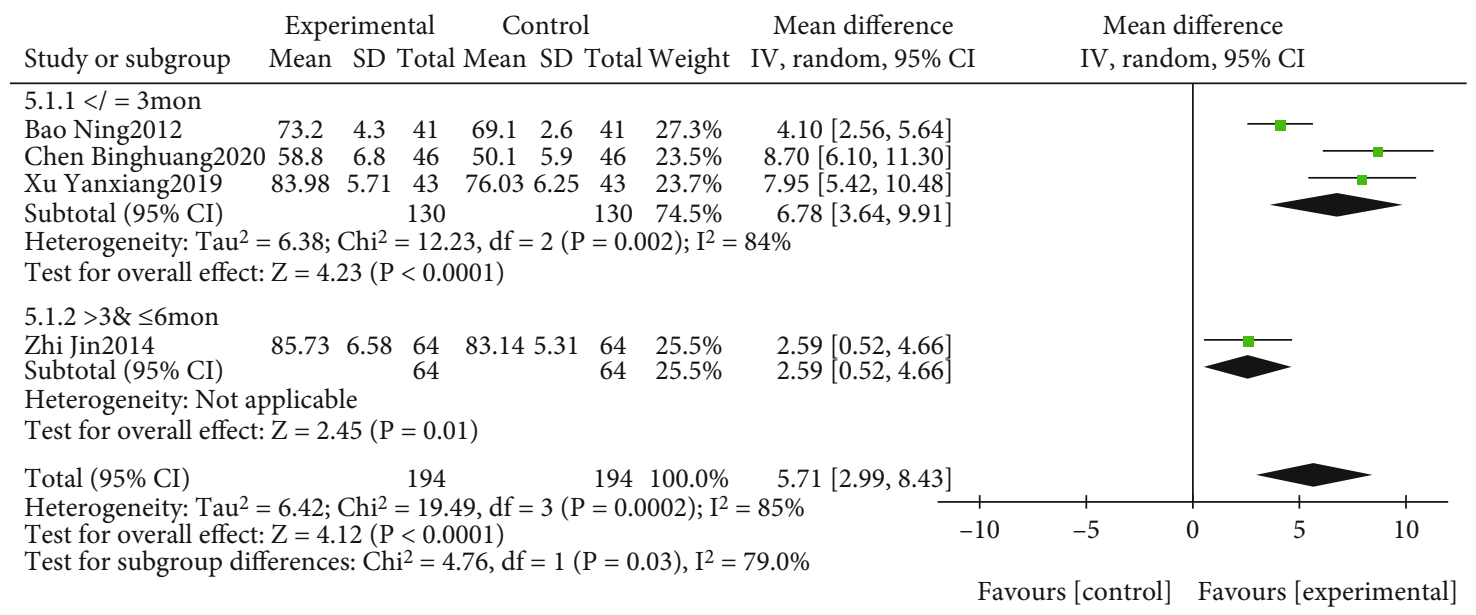

FIGURE 17: Forest plot of comparison: EGb combined with drugs for VCI versus drug treatment alone for VCI on BI levels (different treatments).

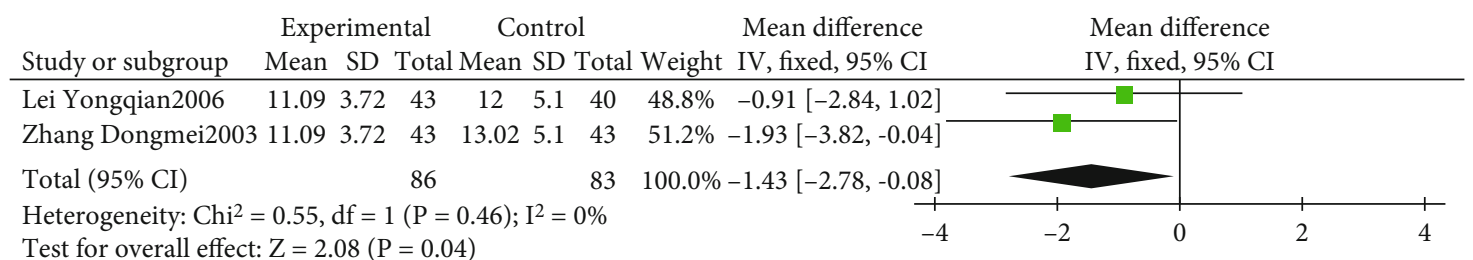

Favours [experimental] Favours [control]

FIGURE 18: Forest plot of comparison: EGb versus drug treatment only for VCI on FAQ levels.

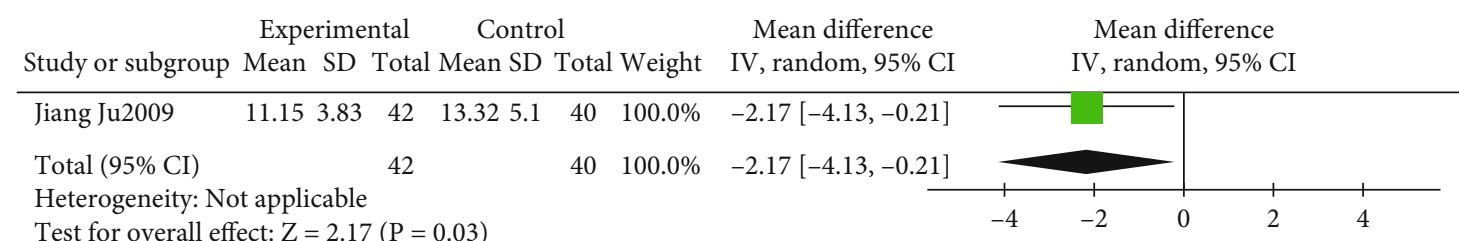

Favours [experimental] Favours [control]

FIGURE 19: Forest plot of comparison: EGb combined with drugs for VCI versus drugs for VCI on FAQ levels.

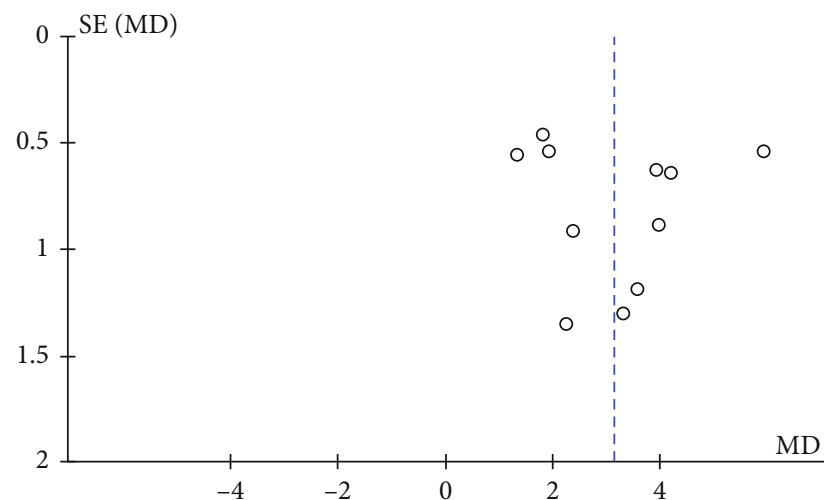

FIgURE 20: Funnel plot of comparison of EGb with drugs for VCI versus drugs alone on MMSE. The horizontal axis shows the mean difference between the estimated effects of EGb with drugs versus drugs alone on MMSE, while the vertical axis shows the standard error of the intervention effect on a reversed scale. reported that no adverse effects occurred during the trial [38, $40,45,56,60]$. The remaining 10 studies $[39,41-44,46-55$, 57-59] reported nine different kinds of adverse events, in which methods for judging adverse events were participant and carer reports, medical notes, or clinical observation or a combination of these. These adverse events were reported in the treatment group and in the control group respectively, except one study which did not mention which group. Six studies reported both groups experienced dizziness/nausea/vomiting/insomnia and diarrhea $[43,44,50,51,54,57]$. Two studies reoported skin rashes [54, 57] and one study reported dry mouth [49] happened in the treatment group, while one study reported gingival bleeding [39] and one study [54] reported elevated transaminase happened in the control group. However, life-threatening adverse effects were not reported in any of the studies. All the details of the reported adverse effects are described in the last column in Table 1. 
3.6. Publication Bias. To explore the issue of publication bias, a funnel plot was constructed in which the standard error of the mean difference was plotted against the mean difference. The funnel plots for the MMSE from 11 studies $[38,40,43,44,46,49-52,54,60]$ that compared EGb with DV versus DV alone suggested publication bias as many of the smaller studies had more positive results (Figure 20).

3.7. Grade Evaluation of Outcomes. GRADEpro software, version 3.6.1, was used to evaluate the quality of evidence of the outcomes. Due to various biases, inconsistencies, inaccuracies, and publication bias, the evidence of certainty ranges from medium certainty to very low certainty, as shown in Table 2.

\section{Discussion}

There is currently no early intervention strategy for cognitive impairment and preventing cognitive decline [61]. The interests of the public and the medical profession in the use of EGb for cognitive impairment and dementia have grown considerably in recent years $[8,61,62]$. There is evidence to support the efficacy of EGb treatment for dementia, and the effect has been found to be dose- and age-dependent [32]. Our analysis supports the efficacy of EGb (in tablet form) for VCI, and it appeared to be well tolerated. This study is a systematic review of the English and Chinese literature to determine the efficacy and safety of EGb for VCI. Twenty-three RCTs including a total of 2019 patients with VCI met the inclusion criteria. The main finding of this review was that EGb treatment appears to be more effective than controls as assessed by various measures of cognitive function, including MMSE, MoCA, HDS, ADL, BI, and FAQ. The evidence of certainty ranges from medium certainty to very low certainty. Although the findings appear positive, the poor methodological quality and clinical heterogeneity of the included studies limit the evidence to support the use of EGb for VCI. In addition, the safety of EGb treatment could not be confirmed because only $65 \%$ $(15 / 23)$ of the studies mentioned safety issues or investigated adverse effects. Due to the limited number of included studies that analyzed safety, we failed to draw a definitive conclusion, which is one of the major issues needing further confirmation. More attention should be paid to both the monitoring and reporting of adverse effects of EGb.

There are some limitations in this study: (1) Only Chinese and English literature was included. All the participants were Chinese, and the administration of EGb was limited to tablets. This could affect the generalization of the results. (2) The data analysis was performed using published trials with positive results, suggesting that trials with negative results may have been missed, which would make the true effect substantially different from the estimate of the effect. (3) The quality of the included trials was generally low, and the certainty of evidence ranged from medium certainty to very low certainty, reducing our confidence in the estimates of the effects. Neither intention analysis nor allocation concealment strategy was mentioned in any of the studies. (4) We put the different kinds of VCI together for each outcome evaluation, although we performed subgroup analysis on the different VCI type as a supplementary analysis. This may not be in line with clinical practice. (5) The treatment courses in most of the included studies were relatively short, and the long-term consequences of EGb treatment for VCI remain unexplored. (6) Clinicians differ in their experience and use of the measurement scales, such as MMSE, $\mathrm{MoCA}$, and $\mathrm{ADL}$. Inconsistent treatment methods in the control group and differences in drug dosage or course may also have an impact on the evaluation of efficacy and safety. The in-homogeneity of the basic supporting treatment may have a confounding effect. Consequently, the results generated from the current review should be interpreted with caution.

Conducting clinical trials in VCI has many obstacles. Clinical outcomes in VCI patients are multifaceted, as they may experience further cognitive decline and may also experience progressive vascular morbidity, mortality, and general deterioration of function $[15,63,64]$. Outcome measures in future trials should include brain structure and function imaging and disease progression determined by macro network diagrams of the brain along with patient-reported outcomes, such as PET (positron emission tomography) and SPECT (single-photon emission computed tomography), measured using a validated $\mathrm{rCBF}$ (regional cerebral blood flow) scale [65-67]. In addition, the 23 included studies contained various types of VCI and different kinds of DV. VCI encompasses a heterogeneous population in terms of cognitive profile and severity of deficits, vascular brain injury, and concurrent neurodegenerative pathology [9]. Thus, the patients should be divided into specific subgroups according to different ages and EGb dosages. It is reasonable to take the most common kinds of VCI with high incidence as future target types to explore the precise benefits obtained from EGb. A long follow-up with long-term outcomes is important to determine the effectiveness and safety of EGb[68]. The safety of EGb is a major concern in clinical practice. Thus, safety monitoring of EGb in pharmacovigilance systems is needed.

Our results are based on published studies, the number of included studies was small, and the quality was poor, which may lead to low credibility of the conclusions. Future research on EGb in VCI should implement higher quality research methodology to limit the potential for bias. More large-scale, multicenter randomized controlled clinical trials on related mechanisms should be implemented in a scientifically designed manner, clinically important outcomes should be selected, and longer treatments and follow-up periods should be used. We recommend that the SPIRIT 2013 and the CONSORT 2010 statement [69-72] should be used as a guideline when designing and reporting RCTs for EGb in the future.

\section{Conclusion}

In summary, in patients with VCI, Ginkgo biloba extract tablets can be taken separately or in addition to other medication. Although the available evidence from the present review supported the efficacy of Gingko biloba extract, 


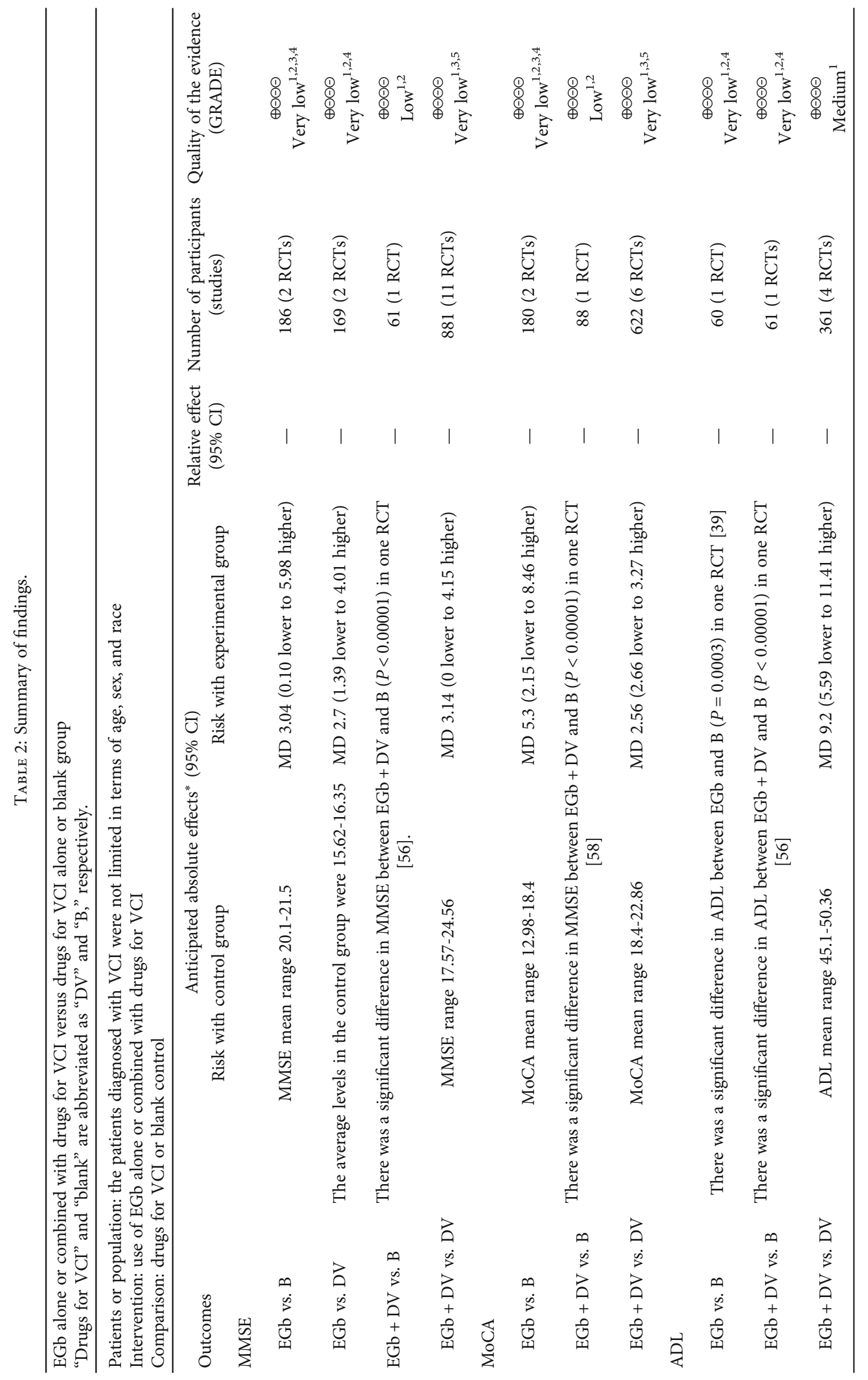




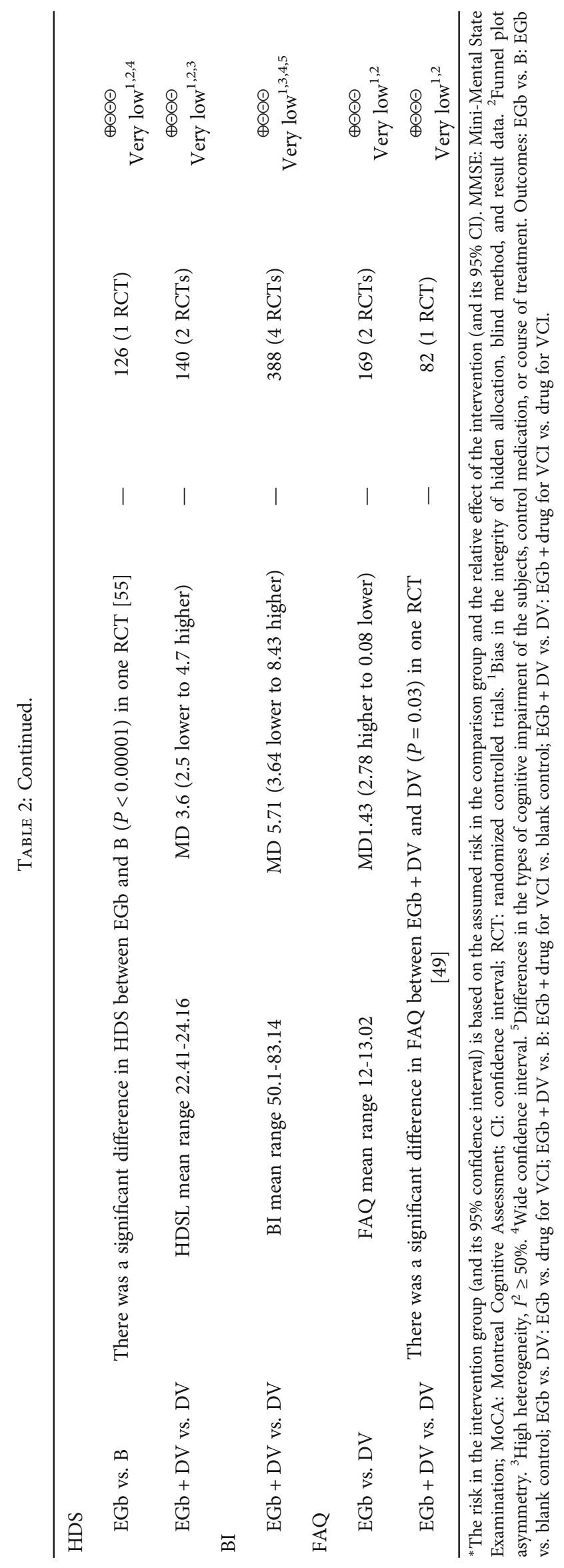


recommendations for its routine use for the treatment of VCI are limited by the poor methodological quality and clinical heterogeneity of the included studies. Nevertheless, we have identified an area that is worthy of further study. Further well-designed rigorous RCTs of Ginkgo biloba extract for VCI are needed.

\section{Data Availability}

All relevant data are within the article and its supporting information files. The data supporting this systematic review and meta-analysis are from published literature and accessible datasets, which have been cited. The original included articles used in this study are available from the corresponding author upon request.

\section{Conflicts of Interest}

The authors declare that the study was conducted in the absence of any commercial or financial relationships that could be construed as a potential conflict of interest.

\section{Authors' Contributions}

Zhan Min and Xing Liao designed the study, conducted the analysis, and drafted the manuscript. Zeng Zixiu and Shen Wei performed the literature selection. Sun Linjuan and Liu Jianxun did the quality assessment. Wang Ying and Han Fuhua performed the data extraction. Shi Jingzi, Zeng Xinyun, and Lu Xiyue did part of the statistical work. Zhang Yunling and Liao Xing critically revised the manuscript.

\section{Acknowledgments}

This study was supported by CACMS Innovation Fund (No. CI2021A00701-3); NATCM TCM Inheritance and Innovation "Hundred-Thousand-Ten Thousand" Talents Project (QiHuang Scholar)-National TCM Leading Personnel Support Program (NATCM Personnel and Education Department [2018] No. 12); the project from the China Center for Evidence Based Traditional Chinese Medicine (No. 2020YJSZX-2); the project of Evidence-Based Medicine of Traditional Chinese Medicine (No. ZZ13-024-3); the Fundamental Research Funds of the Central Public Welfare Research Institutes Grant (No. ZZ13-YQ-075); the National Natural Science Foundation Project of China (No. 81873168); and the National Key Research and Development Program of China (No. 2018YFC1704303).

\section{Supplementary Materials}

Supplementary 1. Appendix 1: checklist of items for the meta-analysis according to PRISMA statement.

Supplementary 2. Appendix 2: supplementary retrieval information: RCTs of Ginkgo biloba Extract in the treatment of VCI.

\section{References}

[1] D. A. Levine and K. M. Langa, "Vascular cognitive impairment: disease mechanisms and therapeutic implications,"
Journal of the American Society for Experimental NeuroTherapeutics, vol. 8, no. 3, pp. 361-373, 2011.

[2] A. Ritter and J. A. Pillai, "Treatment of vascular cognitive impairment," Current Treatment Options in Neurology, vol. 17, no. 8, p. 367, 2015.

[3] R. N. Kalaria, G. E. Maestre, R. Arizaga et al., "Alzheimer's disease and vascular dementia in developing countries: prevalence, management, and risk factors," Lancet Neurology, vol. 7, no. 9, pp. 812-826, 2008.

[4] F. J. Wolters and M. A. Ikram, "Epidemiology of vascular Dementia," Arteriosclerosis, Thrombosis, and Vascular Biology, vol. 39, no. 8, pp. 1542-1549, 2019.

[5] M. Dichgans and D. Leys, "Vascular cognitive impairment," Circulation Research, vol. 120, no. 3, pp. 573-591, 2017.

[6] W. M. van der Flier, W. Flier, I. Skoog, and J. Schneider, "Vascular cognitive impairment nature reviews," Disease primers, vol. 4, 2018.

[7] G. C. Román, "Vascular dementia may be the most common form of dementia in the elderly," Journal of the Neurological Sciences, vol. 203, pp. 7-10, 2002.

[8] P. Sachdev, R. Kalaria, J. O'Brien et al., "Diagnostic criteria for vascular cognitive disorders: a VASCOG statement," Alzheimer Disease and Associated Disorders, vol. 28, no. 3, pp. 206218, 2014.

[9] P. B. Gorelick, A. Scuteri, S. E. Black et al., "Vascular contributions to cognitive impairment and dementia: a statement for healthcare professionals from the American Heart Association/American Stroke Association," Stroke, vol. 42, no. 9, pp. 2672-2713, 2011.

[10] T. J. O'Brien and A. Thomas, "Vascular dementia," The Lancet, vol. 386, no. 10004, pp. 1698-1706, 2015.

[11] G. Davis, N. Baboolal, A. Mc Rae, and R. Stewart, "Dementia prevalence in a population at high vascular risk: the Trinidad national survey of ageing and cognition," BMJ Open, vol. 8, no. 2, article $\mathrm{e} 018288,2018$.

[12] H. Fillit and J. Hill, "The costs of vascular dementia: a comparison with Alzheimer's disease," Journal of the Neurological Sciences, vol. 203-204, pp. 35-39, 2002.

[13] D. S. Knopman, W. A. Rocca, R. H. Cha, S. D. Edland, and E. Kokmen, "Survival study of vascular dementia in Rochester, Minnesota," Archives of Neurology, vol. 60, no. 1, pp. 85-90, 2003.

[14] M.-K. Sun, "Potential therapeutics for vascular cognitive impairment and dementia," Current Neuropharmacology, vol. 16, no. 7, pp. 1036-1044, 2018.

[15] P. Moorhouse and K. Rockwood, "Vascular cognitive impairment: current concepts and clinical developments," The Lancet Neurology, vol. 7, no. 3, pp. 246-255, 2008.

[16] S. T. Pendlebury and P. M. Rothwell, "Prevalence, incidence, and factors associated with pre-stroke and post-stroke dementia: a systematic review and meta-analysis," The Lancet Neurology, vol. 8, no. 11, pp. 1006-1018, 2009.

[17] G. Livingston, J. Huntley, A. Sommerlad et al., "Dementia prevention, intervention, and care: 2020 report of the Lancet Commission," The Lancet, vol. 396, no. 10248, pp. 413-446, 2020.

[18] F. Defeudis and K. Drieu, "Ginkgo biloba extract (EGb 761) and CNS functions basic studies and clinical applications," Current Drug Targets, vol. 1, no. 1, pp. 25-58, 2000.

[19] A. Shaito, D. T. B. Thuan, and H. T. Phu, "Herbal medicine for cardiovascular diseases: efficacy, mechanisms, and safety," Frontiers in pharmacology, vol. 11, 2020. 
[20] Y. Z. Wu, S. Q. Li, X. G. Zu, J. du, and F. F. Wang, "Ginkgo biloba extract improves coronary artery circulation in patients with coronary artery disease: contribution of plasma nitric oxide and endothelin-1," Phytotherapy Research, vol. 22, no. 6, pp. 734-739, 2008.

[21] J. R. Evans, "Ginkgo biloba extract for age-related macular degeneration," Cochrane Database of Systematic Reviews, no. 1, article CD001775, 2013.

[22] G. Yang, Y. Wang, J. Sun, K. Zhang, and J. Liu, "Ginkgo biloba for mild cognitive impairment and Alzheimer's disease: a systematic review and meta-analysis of randomized controlled trials," Current Topics in Medicinal Chemistry, vol. 16, no. 5, pp. 520-528, 2016.

[23] N. Kandiah, P. A. Ong, T. Yuda et al., "Treatment of dementia and mild cognitive impairment with or without cerebrovascular disease: expert consensus on the use ofGinkgo bilobaextract, EGb 761 ${ }^{\circledR}$," CNS Neuroscience \& Therapeutics, vol. 25, no. 2, pp. 288-298, 2019.

[24] F. Tchantchou, Y. Xu, Y. Wu, Y. Christen, and Y. Luo, "EGb 761 enhances adult hippocampal neurogenesis and phosphorylation of CREB in transgenic mouse model of Alzheimer's disease," The FASEB Journal, vol. 21, no. 10, pp. 2400-2408, 2007.

[25] Y. Wu, Z. Wu, P. Butko et al., "Amyloid- -Induced pathological behaviors are suppressed by Ginkgo biloba extract EGb 761 and ginkgolides in transgenic Caenorhabditis elegans," Journal of Neuroscience, vol. 26, no. 50, pp. 13102-13113, 2006.

[26] P. Költringer, W. Langsteger, and O. Eber, "Dose-dependent hemorheological effects and microcirculatory modifications following intravenous administration of Ginkgo biloba special extract EGb 761," Clinical hemorheology, vol. 4, no. 15, pp. 649-656, 1995.

[27] T. Yoshitake, S. Yoshitake, and J. Kehr, "The Ginkgo biloba extract EGb $761^{\circledR}$ and its main constituent flavonoids and ginkgolides increase extracellular dopamine levels in the rat prefrontal cortex," British Journal of Pharmacology, vol. 159, no. 3, pp. 659-668, 2010.

[28] J. Birks, J. Grimley Evans, and Cochrane Dementia and Cognitive Improvement Group, "Ginkgo biloba for cognitive impairment and dementia," Cochrane Database of Systematic Reviews, no. 1, 2009.

[29] S. Gauthier and S. Schlaefke, "Efficacy and tolerability of Ginkgo biloba extract EGb $761{ }^{\circledR}$ in dementia: a systematic review and meta-analysis of randomized placebo-controlled trials," Clinical Interventions in Aging, vol. 9, article 2065, 2014.

[30] H. Amieva, C. Meillon, C. Helmer, P. Barberger-Gateau, and J. F. Dartigues, "Ginkgo biloba extract and long-term cognitive decline: a 20-year follow-up population-based study," PLoS One, vol. 8, no. 1, article e52755, 2013.

[31] S. DeKosky, J. D. Williamson, A. L. Fitzpatrick et al., "Ginkgo biloba for prevention of dementia: a randomized controlled trial," JAMA, vol. 300, no. 19, pp. 2253-2262, 2008.

[32] H.-F. Zhang, "An overview of systematic reviews of Ginkgo biloba extracts for mild cognitive impairment and dementia," Frontiers in Aging Neuroscience, vol. 8, 2016.

[33] M. J. Page, J. E. McKenzie, P. M. Bossuyt et al., "Updating guidance for reporting systematic reviews: development of the PRISMA 2020 statement," Journal of Clinical Epidemiology, vol. 134, pp. 103-112, 2021.

[34] M. Cumpston, T. Li, M. J. Page et al., "Updated guidance for trusted systematic reviews: a new edition of the Cochrane
Handbook for Systematic Reviews of Interventions," Cochrane Database Syst Rev, vol. 10, p. ED000142, 2019.

[35] H. Balshem, M. Helfand, H. J. Schünemann et al., "GRADE guidelines: 3. Rating the quality of evidence," Journal of Clinical Epidemiology, vol. 64, no. 4, pp. 401-406, 2011.

[36] G. Gradepro, Computer program. McMaster University (developed by Evidence Prime) GRADEpro GDT Hamilton (ON), McMaster University (developed by Evidence Prime), 2015.

[37] T. C. Collaboraion, Collaboraion T C. Review Manager (RevMan)[Computer program]. Version [5, 3]. Copenhagen: The Nordic Cochrane Centre, The Cochrane Collaboraion, vol. 5, no. 3, 2014.

[38] F. Li, Y. Xu, C. Li, and H. Nie, "Effect of nimodipine combined with gko leaf on vascular dementia [J]," Nerve injury and functional reconstruction, vol. 1, pp. 30-32, 2013.

[39] F. F. Lu Junfeng and Z. Jinxia, "The effect of gingko leaf on vascular endothelial function in patients with vascular dementia [J]," Chinese modern doctors, vol. 17, no. 58, pp. 16-19+24, 2020.

[40] Z. X. Ma Shuping, "Effect of huperzine A tablet combined with Ginkgo biloba leaf on cognitive ability and hemorheology of patients with vascular dementia," Chinese Community Physicians (General Edition), vol. 6, no. 9, p. 13, 2007.

[41] Z. Z. Meng Xinyong, "Clinical study of Ginkgo biloba combined with piracetam tablet in the treatment of vascular dementia," Chinese Journal of Physician Training, vol. 16, pp. 46-47, 2012.

[42] S. Yifeng, Clinical observation of Ginkgo biloba combined with donepezil in the treatment of vascular cognitive impairment, Nanjing University of Chinese Medicine, 2017.

[43] X. Yanxiang, "Observation on the curative effect of Ginkgo biloba combined with donepezil in the treatment of vascular dementia patients," Medical Innovation in China, vol. 18, pp. 121-124, 2019.

[44] H. S. Zhang Yanna and W. Zhouna, "Clinical effect of donepezil combined with Ginkgo biloba leaf in the treatment of cognitive impairment after cerebral infarction," Chinese Journal of Traditional Chinese Medicine, vol. 7, pp. 1785-1788, 2019.

[45] H. Zhao, C. Xu, Y. Zheng, and J. Su, "Clinical effect observation of ggo biloba leaf combined with donepezil in treatment of vascular dementia [J]," Journal of Practical Medical Technology, vol. 77, pp. 837-839, 2020.

[46] D. B. Zhi Jin P. Jiwen et al., "Observation on the curative effect of Ginkgo biloba combined with huperzine A tablet in the treatment of vascular dementia," Shanxi Traditional Chinese Medicine, vol. 11, pp. 1468-1469, 2014.

[47] L. Xialing, "Effect of Ginkgo biloba leaf combined with nimodipine on 60 cases of vascular cognitive dysfunction without dementia," Chinese Medicine Guide, vol. 17, pp. 187-188, 2015.

[48] L. Yongqian, "Observation on the curative effect of Ginkgo biloba leaf preparation on 43 cases of vascular dementia," Journal of Xiangnan University, vol. 3, pp. 26-27, 2006.

[49] J. Ju, "Ginkgo biloba combined with peilei can treat vascular dementia," Modern Hospital, vol. 8, pp. 56-57, 2009.

[50] H. Xinxia, "Efficacy and safety of donepezil combined with Ginkgo biloba in the treatment of cognitive dysfunction after cerebral infarction," International Medical and Health Review, vol. 24, no. 14, pp. 2105-2106, 2018.

[51] H. Baojun, "Efficacy and safety analysis of donepezil combined with Ginkgo biloba in the treatment of cognitive dysfunction 
after cerebral infarction," Modern drug application in China, vol. 13, pp. 215-216, 2016.

[52] M. B. Guo Zheng, "Clinical observation on 20 cases of vascular dementia treated with donepezil hydrochloride combined with Ginkgo biloba leaves," Chinese Community Physician (Medical Specialty Bimonthly), vol. 11, no. 12, p. 56, 2009.

[53] D. Bin, "Clinical study of Ginkgo biloba leaf combined with nimodipine in the treatment of VCIND," Chinese Tropical Medicine, vol. 2, pp. 210-212, 2014.

[54] B. Chen, Q. Su, Q. Shi, P. Lin, and Y. Znhag, "Treatment of 46 cases of vascular dementia with ginkgo biloba leaves combined with butylphthalide [J]," Fujian traditional Chinese medicine, vol. 51, no. 1, pp. 83-84, 2020.

[55] X. G. Li Zhiguang and W. Haifeng, "Clinical study of Ginkgo biloba leaf combined with sodium cytidine in the treatment of vascular dementia," Journal of New Chinese Medicine, vol. 51, no. 5, pp. 126-128, 2019.

[56] Y. Shuwei, “Clinical observation of Ginkgo biloba combined with XueSaitong in the treatment of cognitive impairment after cerebral infarction," Jilin Medical Journal, vol. 36, no. 17, pp. 3842-3843, 2015.

[57] L. D. Zhang Dongmei, "Observation of therapeutic effect of Ginkgo biloba leaf preparation on vascular dementia," Practical gerontology, vol. 17, no. 4, pp. 197-199, 2003.

[58] F. F. H. Zhang Zejian, "Clinical observation of Ginkgo biloba leaf combined with olacetam capsule in the treatment of mild cognitive impairment after stroke," Grassroots Medical Forum, vol. 23, no. 20, pp. 2815-2817, 2019.

[59] W. J. Zou Tiansheng and H. Shanshan, "Combination of traditional Chinese and western medicine in the treatment of non dementia vascular cognitive dysfunction," JiLin journal of traditional chinese medicine, vol. 33, no. 5, pp. 487-489, 2013.

[60] H. Y. Bao Ning, "Clinical effect of donepezil hydrochloride combined with Ginkgo biloba leaf in the treatment of vascular dementia," Chinese Medicine, vol. 7, no. 8, pp. 965-966, 2012.

[61] O. A. Skrobot, S. E. Black, C. Chen et al., "Progress toward standardized diagnosis of vascular cognitive impairment: guidelines from the vascular impairment of cognition classification consensus study," Alzheimer's \& Dementia, vol. 14, no. 3, pp. 280-292, 2018.

[62] Writing Group of Dementia and Cognitive Impair Group N S, Chinese Medical Association, "Guidelines for the management of vascular cognitive disorders," Chinese Journal of Neurology, vol. 2, no. 44, 2011.

[63] W. M. Van Der Flier, I. Skoog, J. A. Schneider et al., "Vascular cognitive impairment," Nature Reviews Disease Primers, vol. 4, no. 1, pp. 1-16, 2018.

[64] T. J. O'Brien, T. Erkinjuntti, B. Reisberg et al., "Vascular cognitive impairment," The Lancet Neurology, vol. 2, no. 2, pp. 8998, 2003.

[65] X.-N. Zuo and X.-X. Xing, "Test-retest reliabilities of restingstate FMRI measurements in human brain functional connectomics: a systems neuroscience perspective," Neuroscience \& Biobehavioral Reviews, vol. 45, pp. 100-118, 2014.

[66] D. Papo, J. M. Buldú, S. Boccaletti, and E. T. Bullmore, Complex network theory and the brain, The Royal Society, 2014.

[67] M. Xia, J. Wang, and Y. He, "BrainNet viewer: a network visualization tool for human brain connectomics," PLoS One, vol. 8, no. 7, article e68910, 2013.

[68] H. Chen, C. Zhou, M. Yu et al., "The effect of Ginkgo biloba dropping pills on hemorheology and blood lipid: a systematic review of randomized trials," Evidence-based Complementary and Alternative Medicine, vol. 2019, Article ID 2609625, 12 pages, 2019.

[69] X. Liu, L. Faes, M. J. Calvert, and A. K. Denniston, "Extension of the CONSORT and SPIRIT statements," The Lancet, vol. 394, no. 10205, p. 1225, 2019.

[70] S. M. Eldridge, C. L. Chan, M. J. Campbell et al., "CONSORT 2010 statement: extension to randomised pilot and feasibility trials," BMJ, vol. 355, p. i5239, 2016.

[71] K. F. Schulz, D. G. Altman, D. Moher, and CONSORT Group, "CONSORT 2010 statement: updated guidelines for reporting parallel group randomized trials," Annals of internal medicine, vol. 152, no. 11, pp. 726-732, 2010.

[72] S. J. Dutton, “The SPIRIT 2013 statement,” Maturitas, vol. 78, no. 1, pp. 1-2, 2014. 